\title{
An Experimental Investigation of Applying Mica2 Motes in Pavement Condition Monitoring
}

\author{
Jin-Song Pei, ${ }^{1, *}$ Richard A. IVEy, ${ }^{2}$ HungJr Lin, ${ }^{1}$ AAron R. LANDrum, ${ }^{1}$ \\ Colby J. Sandburg, ${ }^{1}$ NAdim A. Ferzli,${ }^{3}$ Timothy King,${ }^{3}$ Musharraf M. Zaman, ${ }^{4}$ \\ HAZEM H. REFAI ${ }^{3}$ AND ERIC C. MAI ${ }^{1}$ \\ ${ }^{1}$ School of Civil Engineering \& Environmental Science, University of Oklahoma, Norman, OK 73019-1024, USA \\ ${ }^{2}$ Department of Industrial \& Systems Engineering, Auburn University, Auburn, AL 36849-2912, USA \\ ${ }^{3}$ School of Electrical \& Computer Engineering, University of Oklahoma, Norman, OK 73019-1024, USA \\ ${ }^{4}$ College of Engineering, University of Oklahoma, Norman, OK 73019-1024, USA
}

\begin{abstract}
Pavement maintenance is vital for travel safety, thus detecting dangerous road conditions in a real-time fashion is desirable. Using an off-the-shelf wireless sensor network to detect such conditions at a low cost poses many challenges. In order to meet these challenges, a Mica2 Mote sensor network is adopted in this study to process and transmit data collected from three external analog sensors. Consequentially, several hardware and software interfaces are developed to complete a pavement monitoring system that uses temperature and moisture presence to detect hazardous road conditions. Surge Time Synchronization is explored in this specific application to enable the wireless sensor network to operate in a low power consumption mode. A fairly simplistic pattern classification algorithm is embedded into the motes to create the smart wireless sensing application. A series of outdoor tests are conducted in this study paying special attention to the survivability of fragile analog sensors in harsh roadway conditions. In this regard, a novel solution called the "Sensor-Road Button"(SRB) is developed and validated experimentally. This is one of several exercises made in this study to enable the application of sensor technologies in intelligent transportation systems (ITS). The size of the wireless sensor network in this study is relatively small, utilizing a total of five motes in order to fully exploit the transmitting range of each mote. Long testing periods (i.e., uninterrupted 12-hour time frames for each period of data collection) add an additional advantage, allowing for the evaluation of the selected wireless sensor network for long-term monitoring using the low power consumption mode under Surge Time Synchronization. Many performance metrics of the adopted small-size, large-interval Mica2 Motes wireless sensor network are revealed in this study through a series of data processing efforts. Results are presented to examine (i) inter-node connectivity and transmitting range, (ii) battery life, (iii) the length of the initial network connection time as affected by methods of setting up tests under practical conditions, (iv) error rate and analysis of different error types (showing the importance of the subsequent data cleansing step), and (v) other network routing properties including the parent time histories for each mote. The results and analysis form a database for future efforts to better understand, appreciate, and improve the performance of Mica2 Motes. This study will thus benefit robust real-world implementation of off-the-shelf sensor network products such as Mica2 Motes in terms of hardware development and data processing.
\end{abstract}

Key Words: Mica2 Motes, wireless sensor network, pavement condition monitoring, structural health monitoring, ITS.

\section{INTRODUCTION}

$\mathbf{P}$ AVEMENT maintenance is vital for travel safety and remains a major issue for transportation

*Author to whom correspondence should be addressed. E-mail: jspei@ou.edu

Figures 1-12 appear in color online: http://jim.sagepub.com agencies nationwide. It is essential to provide warnings of dangerous traffic conditions such as wet and icy pavement in a real-time fashion. This paper presents a preliminary study to apply an off-the-shelf wireless sensor network product, Mica2 Motes (Crossbow Technology, 2006), to address this practical need. A general search (e.g., using GlobalSpec, 1999-2006) on commonly used sensors for roadway safety 
monitoring is conducted and summarized in Pei et al. (2007). The main companies that specialize in this field include Innovative Dynamics Inc. with their IceSight weather information system (Innovative Dynamics, 2000-2006), and Vaisala (Vaisala (2004a, b)) with their road \& runway sensor and surface analyzer. These products provide a benchmark for the proposed Mica2 Motes-based roadway monitoring system. The ultimate functionality of the proposed system should be comparable with the existing systems, while the cost of the proposed system should be reduced drastically for enhanced practical applications. More importantly, an off-the-shelf wireless sensor network product will be adopted as the backbone for this road monitoring system, which has not yet been explored.

Technologies in wireless sensor networks are advancing rapidly, and these emerging technologies inspire engineers to explore new applications that address long-existing and challenging 'old' problems. For example, 'Smart Dust', a low power system that integrates sensing, computing and wireless communication into one product has drawn tremendous attention in recent years (e.g., Mahgoub and Ilyas, 2006). This study in particular is one of the many attempts to use Mica2 Motes (the third generation mote module of the 'Smart Dust' wireless network) with a focus on developing an intelligent transportation system for roadway safety. Three off-the-shelf external sensors suitable for pavement condition monitoring are selected and interfaced to a node of a Mica2 Motes network. An algorithm is further embedded into the node to classify pavement surface conditions into three categories: dry, wet, and icy, based on the measurements taken by these sensors. The processed results are finally transmitted by Mica2 Motes. Many technical issues encountered in this application are addressed, and the performance of the Mica2 Motes wireless sensor network is examined.

To better understand the pros and cons of Mica2 Motes in real-world applications, a literature review is conducted including the works in computer science and pertinent case studies of 'Smart Dust' in the structural health monitoring community within civil engineering. Table 1 summarizes the key findings/metrics in some selected technical publications. In this nonexhaustive list, special attention is paid to network performance (e.g., transmitting range), operational conditions (e.g., antenna height and sampling rate), and application requirements (e.g., the presence of embedded algorithms). The gaps in these existing applications/practices include (i) short transmitting range in the context of civil engineering applications, (ii) lack of considerations for real-world power consumption, (iii) short testing duration that is insufficient for long-term monitoring, and (iv) lack of packaging solutions to protect nodes from accidentally applied loads or undesirable environmental factors.
To bridge the gaps in the applications of 'Smart Dust', this experimental investigation focuses on the following aspects:

Large Transmitting Range: Uniform mote intervals of 25,50 , and 100 feet are tested in outdoor environments. In civil infrastructures, the cost of the monitoring system is a typical constraint, thus the philosophy of 'less is more' is likely to prevail in the deployment of sensors.

Low Transmitting Power: Three options for using Mica2 Motes are available including Surge Reliable, Surge Time Synchronization and Surge Low Power. Surge Time Synchronization is adopted and tested in this study as it allows each mote to be activated from an idle state at a predefined time interval. Surge Reliable is also used initially as a comparison (Ferzli et al., 2006b, a; Pei et al., 2007).

Long Testing Durations: A series of outdoor tests are conducted in this study with a duration of $12 \mathrm{~h}$ or longer.

Effective Packing Solutions: The scope of this study includes the hardening of both the selected sensors and motes to ensure their survivability and performance. Added casing and enhanced antenna detailing validate the recommendation in Crossbow Technologies (2004). Other developed testing accessories are also among the contributions of this study.

In addition to the aforementioned features, an idealized pattern classification algorithm is used in this study to categorize pavement surface conditions based on three types of measurements, as shown in Figure 1(a).

\section{DEVELOPMENT DETAILS}

A gap often exists between available sensor technologies and the harsh real-world environments in which they are meant to be deployed/implemented. To bridge this gap, careful engineering design and novel solutions are necessary. In this study, it is not trivial to implement the selected sensor products and Mica2 Motes in a roadway environment to perform real-time monitoring. The efforts made in this study can be considered an important step towards developing full-scale commercial products. The prototypes of products developed here could be refined and improved through continued research.

\section{Selected Sensors and Developed Sensor-Road Button}

Based on the proposed algorithm to detect the existence of ice, three types of external sensors were selected to interface with one Mica2 Mote. 


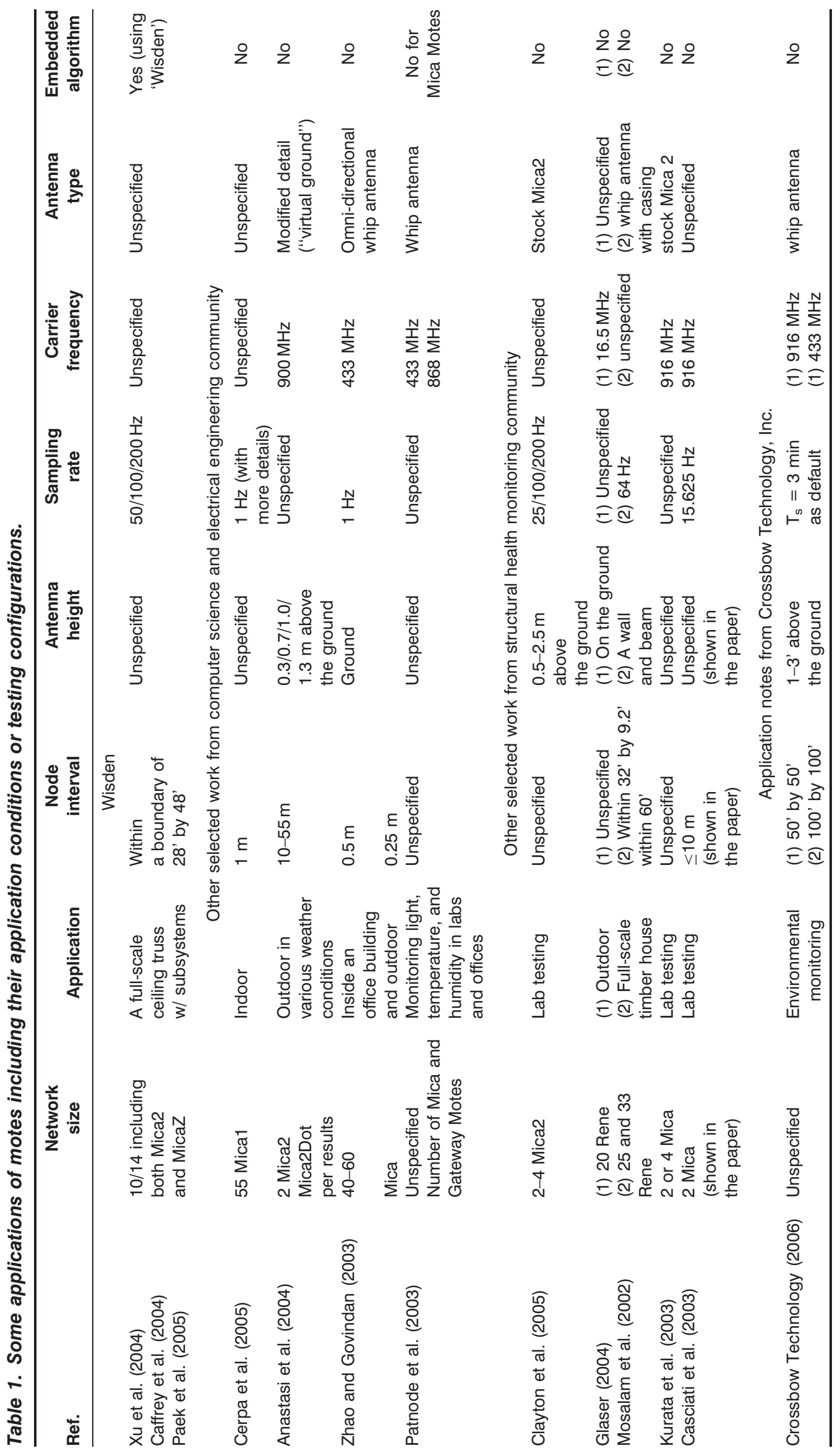




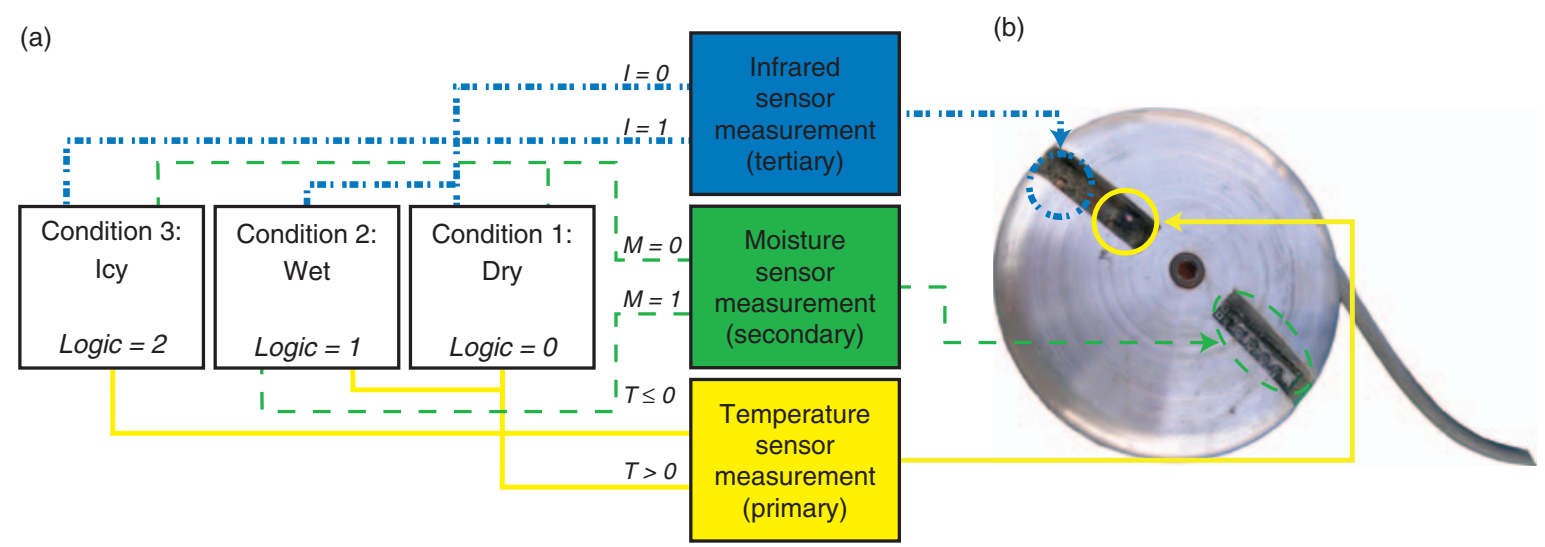

Figure 1. (a) Proposed algorithm to classify road surface conditions, where $T, M$, and I denote the converted readings from the selected thermistor, leaf, and infrared sensors (with yellow, green dashed, and blue dash-dotted lines), respectively. Specifically, $T$ is the measured temperature in degrees Celsius. $M=1$ denotes a high voltage output of the moisture sensor indicating the presence of moisture. $I=1$ denotes a high voltage output of the infrared sensor indicating the presence of ice. The determination of the decision boundaries for high and low voltages is presented in the section 'Sensor-mote interface and embedded algorithm'. (b) A photograph showing the top view of the three sensors on the Sensor-Road Button (SRB).

Temperature, moisture, and infrared sensors were selected for this study considering their cost, power consumption, size, and durability. In detail, the sensors selected included a $10 \mathrm{~K} \Omega$ thermistor (Vishay, 2004), a leaf sensor used to detect the conductivity of wet pavement and thus the existence of free moisture (Davis Instruments, 2002), and a reflective object sensor composed of an infrared emitter and an infrared receiver of the same wave length (Fairchild Semiconductor, 2000).

Calibration tests were performed on the selected thermistor using a freeze-thaw chamber. In addition, all three sensors were combined and tested to successfully validate the proposed pattern classification algorithm shown in Figure 1(a) utilizing a thawing process, the result of which is presented in Pei et al. (2007) and Ferzli et al. (2006b, a). These test results also provided decision boundaries required for the pattern classification algorithm in Figure 1(a).

For the three selected sensors, a rugged casing (i.e., sensor casing) is needed to keep these sensors active and functioning properly in the specified application. After considering many design options pertaining to locations of the sensors and shapes of the casing (Pei et al., 2007), surface road buttons were deemed as the best choice (Traffic Buttons, 2005). An evaluation of these existing products and design led to a product called 'Sensor-Road Button' (SRB) invented in this study.

Figure 1(b) shows the final version of the SRB developed in this study. In general, this metalware has an upper and a lower piece connected by a stainless steel socket-head cap screw. The dome-shaped upper piece is 4 inch in diameter and 0.5 inch in height, which intrudes above the ground. The lower piece is of cylindrical shape with a diameter of 4 inches and a height of 1(5/8) inches. The top view of the upper piece is shown in Figure 1(b), while the lower piece provides protection from the ground for the sensors and a portion of the sensor wires.
Suspended on one side of the SRB is the leaf wetness sensor, while the thermistor and infrared sensor are located on the other side. Instead of using a piece cut from a commercial leaf sensor as was done in earlier versions of the SRB (Pei et al. (2007)), electronic solders were deposited directly onto a small piece of prototyping board to form a home-made sensor (as was done with the leaf wetness sensors). Adjacent metals were made close to each other to form a finger-like shape having a 1(1/8) inch width. This two-part design not only provides sufficient protection, but also provides an easy access to the sensors. As documented and analyzed in Pei et al. (2007), the design of this academic SRB unit provides sufficient protection for the sensors. This can be seen in several survivability tests using a 9.4-kip forklift.

\section{Mote Casing and Improved Antenna Detail}

The design of the mote casing is related to the design of the sensor casing. While the three selected external sensors can be placed on the surface of a roadway shoulder using a sensor casing, the mote and antenna (both are connected to the sensors through a cable) should be stored in a basket-like structure called the mote casing. The mote casing is elevated to provide good signal reception, long transmitting range and, to some degree, the prevention of vandalism. In this study, the heights vary between 33 inches, 6 , and 9 feet. The former can be thought of as the height of a typical median-size barrier wall (Oklahoma Department of Transportation, 1999) and conveniently achieved using timber post. The latter two heights allow for clearance of most traditional cars and trucks and are conveniently achieved by a steel post set up along a roadway. The final mote casing design is shown in Figure 2.

According to the recommendations in Crossbow Technologies (2004), optimizing the antenna 
(a)

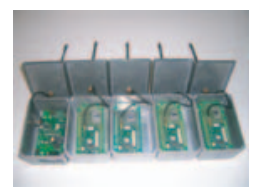

(b)
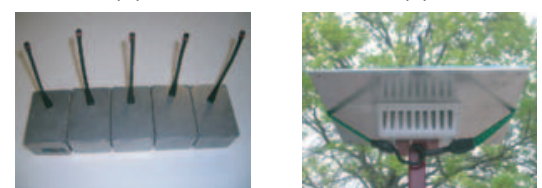

Figure 2. (a) and (b) represent different views of the packaged Motes without the plates, and (c) shows views of the added antenna plate being installed and used.

polarization is a key factor for transmitting range. Polarization refers to the spatial orientation of the lines of flux in an electromagnetic field. An antenna horizontal to the ground is horizontally polarized, while an antenna perpendicular to ground is vertically polarized. Optimizing the antenna polarization means keeping all antennas aligned in a horizontal or vertical position. To achieve the maximized transmitting range, the antennas should be oriented with polarization whenever possible (Milligan, 2005). In the results reported here, all antennas have been vertically aligned, which is made possible by adopting the mote casing with a replacement antenna.

Precisely following the detailed recommendation in Crossbow Technologies (2004), permanent studmount quarter wave whip antennas (model ANT433-PW-QW) from Linx Technologies, Inc. (Linx Technologies, 2006), shown in Figure 2(b) and (c), are adopted to replace the built-in antennas with the original packaging of motes. The Linx antenna is able to remain vertically aligned as it is stud mounted on to the lid of the mote casing (Figure 2(b)). This proves to be a better option to ensure proper alignment of the antenna during testing. The antenna is connected to a mote through an MMCX coaxial connector (Amphenol RF, 2003-2005). However, this connection has caused another challenge to the robustness of the system. In this experimental study, hardware connection problems were caused by damaged connectors due to heavy use, especially during frequent plugging and unplugging of the antennas from the motes, as required by different configurations.

An aluminum casing (Hammond Manufacturing, 2006) shown in Figure 2(a) and (b), was chosen to house the mote, with the antenna extending out of the top covering. The casing's overall dimensions are $2(1 / 4)$ $\times 3(1 / 4) \times 4(3 / 4)$ inches $(H \times W \times L)$. Theoretically, the casing could act as a reflecting ground and simulate a dipole effect for the antenna. However, the surface area of the selected aluminum casing is not large enough to enable the dipole effect because of the required half wave length in each direction (Milligan, 2005).

To produce a rigorous condition for the dipole effect, the square aluminum plate, shown in Figure 2(c), was adopted on the top of the existing aluminum casing lid. The plate measures $14 \times 14 \times(1 / 8)$ inches. To fit this plate properly to the mote casing described above, an opening was drilled through the center of the lid in the mote casing to retain the balance of the entire structure of the mote casing and the plate. Moreover, a bungee cord was utilized to stabilize the plate on the top of the mote casing when the entire unit was placed inside a plastic basket for long outdoor testing. This introduced opening on the lid of the casing caused a potential problem as rain water could easily seep into the casing through the gap between the edge of the opening and the body of the antenna.

Solutions developed in this study to solve this problem are proven to be effective (Pei et al., 2007). An incident during one of the tests accidently validated the effectiveness of the improved detailing of mote packaging. During 2 days of testing, a mote was accidently exposed to a rain event which produced 0.25 inches of rain (http://www.srh.noaa.gov/). Despite the exposure, the aforementioned measures taken to protect the motes proved effective because no damage to the mote occurred. Another successful rain protection case occurred when light showers fell during the final half-hour of testing on September 1, 2006 (see Figure 12(a) for the readings from the sensors on the SRB). All of the motes were immediately retrieved once the test was terminated and no damage to the motes occurred.

\section{Sensor-Mote Interface and Embedded Algorithm}

The efforts to interface the three selected sensors to a mote consists of both hardware and software implementations. As presented previously, an integrated unit with the sensors and a structure mimicking a road button was created in this study (the SRB). An MTS101 prototyping board from Crossbow Technologies, Inc. was used to ease the connection of these sensors to a mote. The MTS101 is an expansion of a mote that permits easy access to input/output pins. Each Mica2 Mote has a 51-pin Hirose connector that connects to various input/output $(\mathrm{I} / \mathrm{O})$ pins within the CPU (Maurer, 2003). In this study, three A/D inputs are used for the three selected sensors. All three sensors were powered by the mote due to their low power consumption need; a simple circuit was designed to furnish the correct voltage to power these sensors. A shielded wire (Figure 1(b)) was used to connect the SRB to the MTS101 board, which protected the circuitry from external interferences throughout this study. Three power control outputs were also used as reference voltage to the three sensors. The sensors were in the off position when not in use to fulfill the low power consumption requirements and were effectively turned on when in use.

Using Surge Time Synchronization (Brewer et al., 2004), the software implementation involves four inputs and three outputs, as shown in Figure 3. As detailed in Pei et al. (2007), four subsequent states 


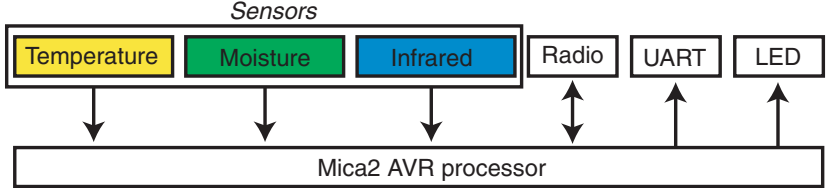

Figure 3. System context diagram.

related to this software design include idle, retrieve and broadcast data, receive and process sensor data, and send data. Upon receiving 'sync bits,' a programmed mote leaves the first state and goes through the last three states one by one. Once the send data (final state) is performed successfully, the mote returns to the idle state. Data control flow and task architecture are implemented in this study. Various subsystems are developed under TinyOS including main program, sensor interface, embedded algorithm and radio transmission/reception.

For the embedded algorithm, three measurements are received from the selected sensors at each time instance. These sensors are integrated with the aforementioned SRB, which is mounted to a selected location on the surface of the road. The algorithm is designed to process these measurements in real-time to classify the road surface conditions. As shown in Figure 1(a), the temperature, leaf wetness, and infrared sensors are the primary, secondary, and tertiary sensors, respectively. Three numbers are assigned to categorize the pavement conditions; 0,1 , and 2 represent for dry, wet, and icy conditions, respectively. The embedded algorithm directly operates on the raw readings of the temperature, moisture, and infrared sensors. As mentioned previously, laboratory testing provided the calibration relationship for the thermistor and decision boundaries for the other two sensors (i.e., how high and low voltages are differentiated as illustrated in Figure 1(a)). By further considering the 10-bit resolution of the onboard analog-to-digital converters (ADCs), the values of 500 and 800 (i.e., voltage values of 1.613 and 2.581, respectively) were selected as decision boundaries for the leaf wetness and infrared ice sensors, respectively.

A user-friendly LabVIEW graphical user interface (GUI) was developed to collect and display the data from the base station wired to a laptop. The GUI retrieved data over the UART (serial) channel on the base station and filtered the data to display the following: the readings of time stamp, the voltage outputs of three selected sensors, battery voltage, sequential number, node ID, parent ID of the node, and the logic result of the detection algorithm. This GUI is activated by a LabVIEW virtual instrumentation file. After data collection is complete, all received data is then saved into a set of three text files (raw, formatted, and error data files) that will be discussed further in the section 'Data cleansing and processing".

\section{DETAILS OF OUTDOOR TESTING}

\section{Testing Environment and Configuration}

All tests using Mica2 Motes were carried out in an open and quiet area in the vicinity of the Fears Structural Engineering Laboratory (abbreviated as Fears Lab hereafter) on the south campus of the University of Oklahoma as shown in Figure 4. Testing environment, especially the existence of building materials, the condition of traffic flow, and the weather during testing, are among the factors that affect the performance of wireless sensor networks (Shankar, 2002; Mark and Zhuang, 2003; Pei et al., 2008). As shown in Figure 4, the buildings are low-rise and sparse. The buildings in the surrounding areas are slightly more elevated than the lab. All the tests in this study were conducted either on actual roads or inside the fence around Fears Lab. Vehicle traffic was light during the daytime and extremely light during the night when these tests were conducted. Temperatures varied from approximately 20 to above $100^{\circ} \mathrm{F}$ for all the outdoor testing that started in May 2005 and ended in September 2006. A bulk of the data was collected under dry conditions, although there were a few times when tests were conducted during moist and/or raining conditions to examine the robustness of the developed packaging solution. Moist and rainy conditions were also beneficial in studying the performance of the selected wireless sensor network under undesirable conditions (for wireless communication). For essentially the same testing environment, a sperate study by some of the authors shows that the foliage condition does not play a significant role (Pei et al., 2008). Therefore, trees are not indicated in Figure 4.

The outdoor testing evolved four separate stages as detailed in Pei et al. (2007). Surge Reliable was tested in Stage I and Surge Time Synchronization was first modified and tested in a series of daytime tests in Stage II. In general, the test results were discouraging with poor and sometimes no conductivities between the nodes (Ferzli et al., 2006b, a), which prompted extensive trouble shooting and improvement in testing apparatus and procedures (see section 'Mote Casing and Improved Antenna Detail'). In particular, optimizing antenna polarization became understood as a key aspect in trouble shooting, as recommended in Crossbow Technologies (2004). Stages III and IV, when the performance of Surge Time Synchronization was tested, are focused in this article.

Stage III-Late Spring and Early Summer 2006: The Surge Time Synchronization was further tested with improved testing apparatuses during a series of 'overnight tests,' each lasting for at least $12 \mathrm{~h}$, typically from $8 \mathrm{pm}$ to $8 \mathrm{am}$ of the next day. The testing venue was inside the secured 


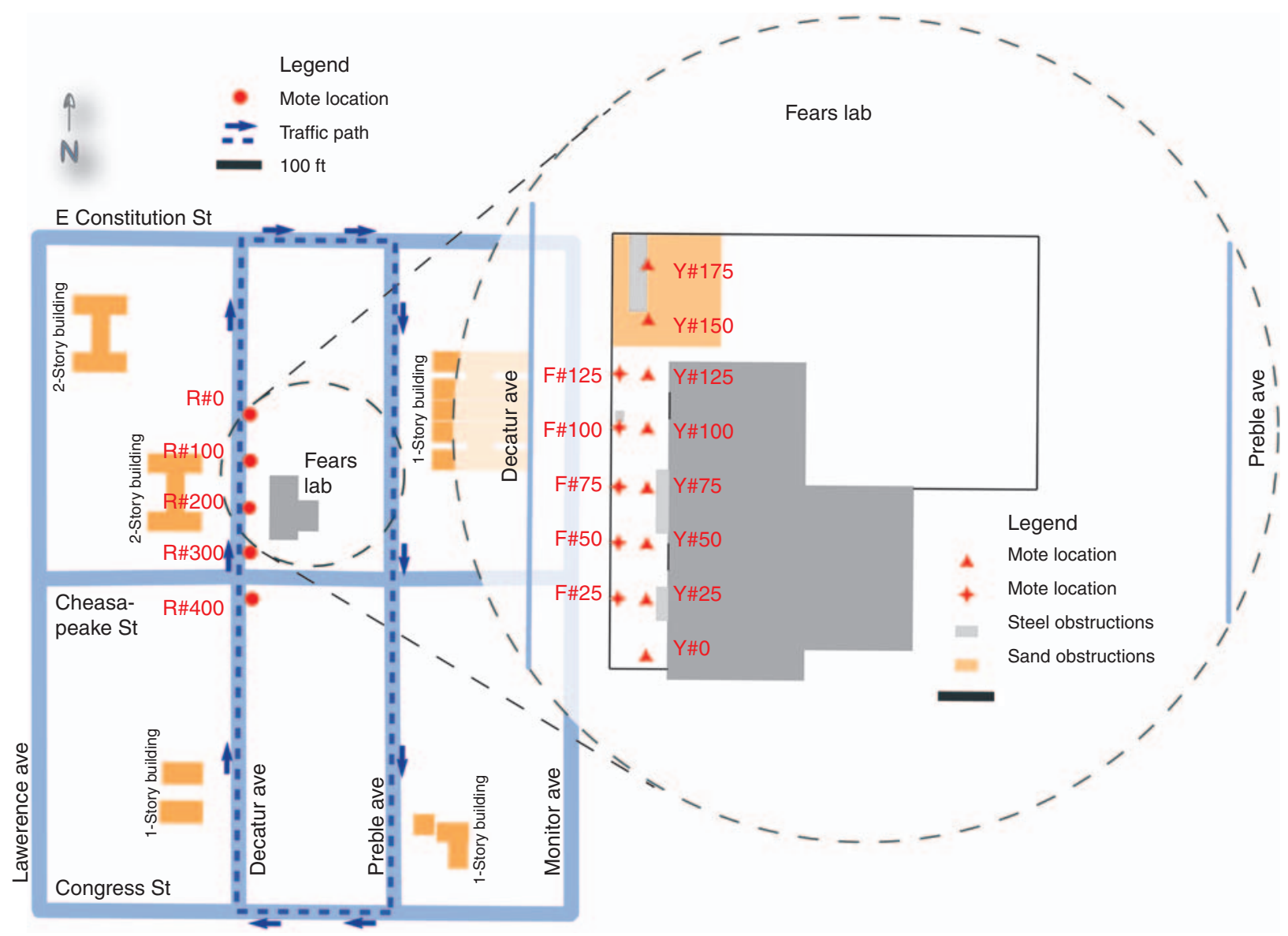

Figure 4. A map showing testing environments and various testing configurations in this study. In some tests at Stage I, artificial traffic interference was introduced following the driving route shown in the figure. The results collected using Surge Reliable are presented in Pei et al. (2007) and Ferzli et al. (2006 a,b)

yard at Fears Lab (see the node locations marked with ' $Y$ ' in Figure 4), and along the fence (see the node locations marked with ' $F$ ' in Figure 4).

Stage IV: Mid Summer to Early Fall 2006: The Surge Time Synchronization was used in a series of 'road tests' along Decatur Avenue with a Sensor-Road Button (SRB) interfaced with one of the network nodes. A typical testing duration for this set of tests was $12 \mathrm{~h}$ from noon to midnight. The node locations are marked with ' $R$ ' in Figure 4.

Panoramic photographs of typical testing environments are shown in Figure 5(a) and (b) for Stages III and IV, respectively to further illustrate the testing environments (which, in turn, affect the performance of wireless communication). These pictures are taken along Decatur Avenue facing east.

The phrase 'overnight testing' is used here to describe the tests that were carried out at Stage III. It means testing the network for long hours in an uninterrupted and unmanned manner. As proven by the data analysis, the $12 \mathrm{~h}$ time window adopted here is sufficiently long to allow the nodes to connect with each other.
The unmanned style (except for test setup and closeup) is labor effective. The nighttime hours were used to minimize the uncertain effects from human activities and rotary machines inside Fears Lab as well as traffic interference in the vicinity of the Fears lab. Therefore, the overnight test results can be considered as the best possible scenario (i.e., an upper bound) to evaluate the performance of the wireless sensor network. Another advantage of running these long-hour outdoor tests is the convenience of examining the impact of ambient weather conditions to the packaging of the hardware. For a real-world implementation of any wireless sensor network, the rigidity of the product must, at least, sustain this level of physical abuse.

It should be noted that the selected secured area for overnight testing was never meant to be perfect in terms of maximizing signal transmission. As illustrated in Figures 4 and 5: (i) the testing venue was fairly congested with various equipment and construction materials as in a typical university structural engineering laboratory, and (ii) there were obtrusions caused by the stored construction materials (mainly steel products). The existence of these materials was expected to cause data loss as studied by some of the authors in a 
(a)

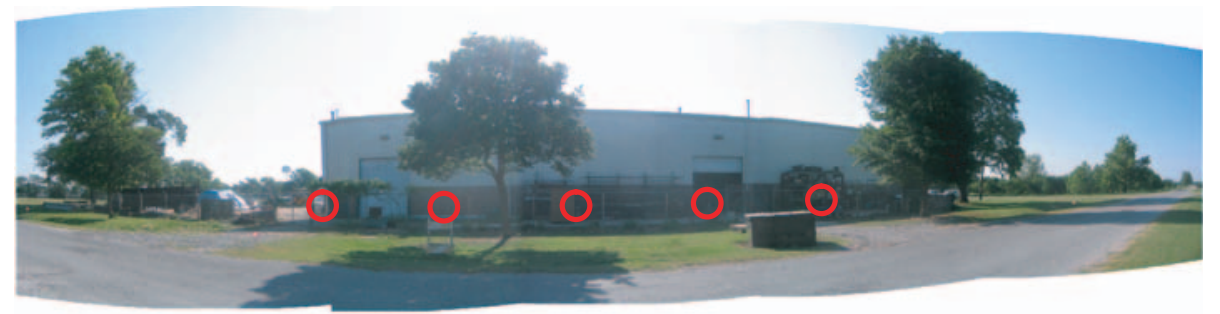

(b)

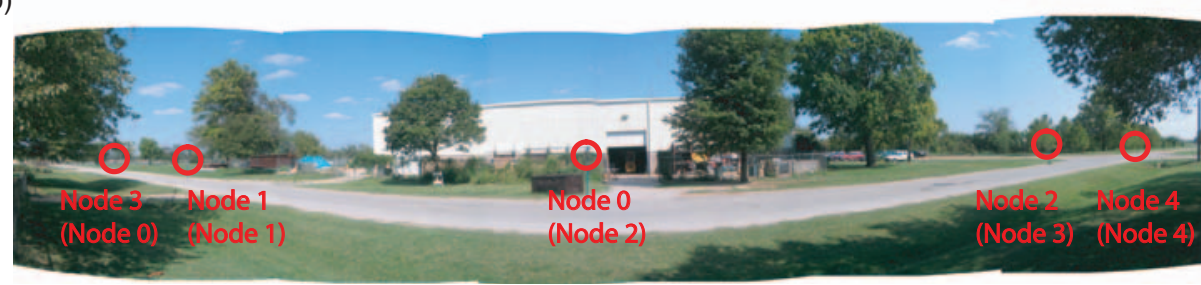

Figure 5. Panoramic pictures of (a) one of the 'overnight tests' at Stage III when mote interval is 25 feet shown in the picture, and (b) one of the 'road tests' at Stage IV when mote interval is 100 feet throughout. The locations of the motes are circled in red.

previous study on a similar transmitting environment (Pei et al., 2008). As one could not realistically expect a perfect line-of-sight (LOS) condition in all real-world applications, such a crowded environment was not disqualified from being adopted as a test bed. By varying several key factors (see below) that would potentially affect the packet delivery performance, meaningful analysis of network performance was made possible by utilizing the available testing environment.

There were many common features at both Stages III and IV. The mote alignment was a chain-like straight line with Node 0 located at one end or in the middle of the chain. The interval between all motes (including Node 0) was made uniform. Due to space constraints, two different intervals ( 25 and 50 feet) were adopted at Stage III. At Stage IV, however, the interval remained 100 feet throughout. The height of the motes was varied at both Stages III and IV. Timber posts of 33 inches in height and steel posts whose heights could be conveniently adjusted between 6 and 9 feet were utilized. In addition to mote alignment, interval, and height, the decision to include or exclude aluminium plates to ensure dipole effect was another parameter to examine at Stage III. A sampling rate of $0.1 \mathrm{~Hz}$ (i.e., one sample every $10 \mathrm{~s}$ ) was used at both Stages III and IV. This sampling rate might be too fast for a long-term monitoring application; however, the large amount of data collected at this elevated sampling rate made these tests an 'accelerated' means to evaluate the performance of Mica2 Motes using Surge Time Synchronization. The most distinctive differences between Stages III and IV include how Node 0 was powered and whether an SRB (i.e., the external sensors) was connected to a node during a test. At Stage III, Node 0 was powered by a wall socket and no SRB was connected to any mote. At Stage IV, however, all motes including Node 0 were powered by batteries and an SRB was connected to one node during each test.

\section{Testing Procedure}

The evolution of outdoor testing was a learning process for the authors. Various difficulties were encountered as the experimental study progressed from one stage to the next. As a consequence, solution strategies were developed to package the selected external sensors, package the motes, and troubleshoot the hardware to better enable outdoor long-hour tests (see section 'Development details' and Pei et al., 2007). In addition, the testing procedure was established in this study when conducting a typical 'road test' at Stage IV.

1. All motes were checked to ensure their proper operation. Each mote was checked for sufficient battery power for the test, based on the data collected from the previous test. The red and black power wires on each mote were also checked, along with the antenna, to ensure proper connections.

2. Secondly, a quick test was conducted in an airconditioned chamber inside Fears Lab to ensure proper connection of all motes under the setting that would be used for the scheduled road test. The motes were placed in the same casings and connected to the same antennas (and the same aluminum plates, if applicable) that would be used in the future 'road test.' The mote intervals were fairly random but remained close. Node 0 was connected to the laptop, and a LabVIEW GUI interface was run to collect data (Pei et al., 2007; Ferzli. et al., 2006b, a). Once the program was running, all motes were turned on. If all motes connected within approximately 
30 min of running the program, then they were turned off and the actual field test could begin. However, if they had not all connected, then a set of troubleshooting steps were pursued.

3. In order to conduct the field test, a laptop was installed inside a timber house that was dedicated to field data acquisition, the selected posts (steel or timber) were placed in their proper positions, and each mote was placed in the basket of a post according to the desired test setup. All of the motes were secured using a bungee cord that was wrapped around the aluminum plate, above the mote casing and basket on top of the post as shown in Figure 2(c). Once the motes were secured, Node 0 was then connected to the laptop through a serial cable and the LabVIEW program was launched as described earlier. Node 0 was then turned on. Once Node 0 was properly connected to the laptop (usually within seconds), all of the other nodes were turned on beginning with Node 1 and ending with Node 4.

4. When at least $12 \mathrm{~h}$ had passed, the test was terminated by stopping the LabVIEW program and saving the appropriate files. For each test, a raw data file, a formatted data file, and an error file were saved. Once the program was terminated, each mote was turned off, and all of the testing equipment was taken down.

\section{DATA CLEANSING AND PROCESSING}

Reliability of wireless data transmission is among the major technical challenges in wireless communication (e.g., Donoho, 2003). Data transmitted through a wireless sensor network can have some unique 'glitches' as compared with that collected from cablebased data acquisition systems (e.g., Pei et al., 2008). In the areas of structural health monitoring and intelligent transportation systems, understanding the issues in data processing and interpretation when employing wireless sensor networks is of great importance in both research and practice.

Efforts were made to examine and clean (while off-line) the data collected by Mica2 Motes using the aforementioned hardware and software developed in this study (see section 'Development details'). A computer scientist would decipher the inner workings of Mica2 Motes and improve their functionality and performance. Here, as an end user, the focus is to reveal the data challenges imposed by a Mote2 Motes-based data acquisition system that is adapted from its generic platform (Crossbow Technologies, 2004) and overcome these data problems through off-line data processing. The perspective of this study could, at least, directly lend experience to other end users.

\section{Format of Data Collected by Mica2 Motes using Surge Time Synchronization}

Every set of data collected by the GUI using Surge Time Synchronization is stored into three files: raw data, formatted data, and an error $\log$ as described in the section 'Testing procedure'. Many explanations can be given to the structure, meaning, and inner-workings of these data files. From an end-user's perspective, formatted data files have complete information organized under various categories and thus are the ones to be cleansed and processed. As illustrated in Table 2, there are a total of nine columns in a formatted data file. Row-wise, each line in this file can be considered as one packet, the smallest unit transmitted by the radio of Mica2 Motes nodes. These lines are organized in a chronologically ascending order, i.e., they are sorted according to time stamp in the format of yymmddHHMMSS.FFF. The time stamp is the time when the base station receives a packet from a node in the network. Once a packet comes in from a node whose ID is recorded in Column 8, the base station sends it to the laptop and the time stamp is assigned and placed in the packet. Sequential number are the numbers of packets being sent out from the base station. If the GUI is turned on first and then Node 0 is powered on, the sequential numbers will start from 1 . When this order is reversed, the sequential numbers may start from a number greater than 1. More details can be found in Pei et al. (2007).

\section{Typical Data Problems Associated with Surge Time Synchronization}

From the perspective of an end user of Mica2 Motes, this subsection further details problems associated with the formatted data files. These problems, which may or may not be 'problems' from the perspective of a computer scientist, are identified and classified entirely for the need of data cleansing and processing. Following the exploration of Mica2 Motes, the authors envision additional improvements made in future studies to directly address some of these problems to some extent before they propagate into the collected data. Overall, there are two types of problems associated with collected data in this study outlined as follows:

Lost Data: Data loss results from the reliability issue of wireless communication. To minimize the latency in data transmission, this study does not include the use of a 're-try' or 're-send' option. Lost data must be identified and the data loss rate (or its complement data reception rate) should be estimated from collected data. As experienced in this study, this problem is always 'hidden,' which means that one must detect the existence of data loss by mining the data. For example, 
Table 2. A typical format of formatted data using Surge Time Synchronization. The sample data is extracted from the results of a test conducted on September 14, 2006. The bold line is related to lost data as explained later in section 'Typical data problems associated with surge time synchronization'. The number 126 stands for the laptop.

\begin{tabular}{|c|c|c|c|c|c|c|c|c|}
\hline $\begin{array}{l}\text { Time stamp } \\
\text { (yymmddHHMMSS.FFF) } \\
\text { (1) }\end{array}$ & $\begin{array}{l}\text { Ice } \\
\text { (volt) } \\
(2)\end{array}$ & $\begin{array}{l}\text { Leaf } \\
\text { (volt) } \\
(3)\end{array}$ & $\begin{array}{c}\text { Temperature } \\
\text { (volt) } \\
\text { (4) }\end{array}$ & $\begin{array}{c}\text { Battery } \\
\text { (millivolt) } \\
(5)\end{array}$ & $\begin{array}{l}\text { Sequential } \\
\text { no. (no unit) } \\
(6)\end{array}$ & $\begin{array}{l}\text { Parent ID } \\
\text { (no unit) } \\
(7)\end{array}$ & $\begin{array}{l}\text { Node ID } \\
\text { (no unit) } \\
(8)\end{array}$ & $\begin{array}{c}\text { Logic } \\
\text { (no unit) } \\
\text { (9) }\end{array}$ \\
\hline 060914122122.621 & 0.834668 & 0.644531 & 1.015137 & 3178.558376 & 165 & 0 & 2 & 1 \\
\hline 060914122122.842 & 0.750879 & 0.72832 & 0.98291 & 2789.202673 & 193 & 126 & 0 & 1 \\
\hline 060914122131.274 & 0.786328 & 0.625195 & 0.986133 & 3025.004831 & 161 & 0 & 3 & 1 \\
\hline 060914122132.776 & 0.750879 & 0.741211 & 0.966797 & 2783.004444 & 194 & 126 & 0 & 1 \\
\hline 060914122140.618 & 0.786328 & 0.612305 & 0.986133 & 3025.004831 & 162 & 0 & 3 & 1 \\
\hline 060914122141.730 & 0.831445 & 0.621973 & 1.011914 & 3178.558376 & 167 & 0 & 2 & 1 \\
\hline
\end{tabular}

Table 3. Four segments of data sorted according to individual nodes to illustrate the first subtype of erroneous data. They are extracted from the results of a test conducted on August 10, 2006. Lines in bold contain erroneous data. Note that the embedded algorithm contained an error at that moment, thus no correct logic result was collected at Node 4 (where the SRB was connected).

\begin{tabular}{|c|c|c|c|c|c|c|c|c|}
\hline $\begin{array}{l}\text { Time stamp } \\
\text { (yymmddHHMMSS.FFF) } \\
\text { (1) }\end{array}$ & $\begin{array}{l}\text { Ice } \\
\text { (volt) } \\
(2)\end{array}$ & $\begin{array}{l}\text { Leaf } \\
\text { (volt) } \\
(3)\end{array}$ & $\begin{array}{c}\text { Temperature } \\
\text { (volt) } \\
\text { (4) }\end{array}$ & $\begin{array}{c}\text { Battery } \\
\text { (millivolt) } \\
(5)\end{array}$ & $\begin{array}{l}\text { Sequential } \\
\text { no. (no unit) } \\
(6)\end{array}$ & $\begin{array}{l}\text { Parent ID } \\
\text { (no unit) } \\
(7)\end{array}$ & $\begin{array}{l}\text { Node ID } \\
\text { (no unit) } \\
(8)\end{array}$ & $\begin{array}{c}\text { Logic } \\
\text { (no unit) } \\
\text { (9) }\end{array}$ \\
\hline 060810144404.667 & 0.802441 & 0.641309 & 1.002246 & 3115.303483 & 740 & 0 & 1 & 1 \\
\hline 060810144414.763 & 1.65 & 1.65 & 0.428613 & 250470.4 & $1,048,320$ & 1017 & 1 & 113 \\
\hline 060810144416.815 & 0.802441 & 0.641309 & 1.002246 & 3115.303483 & 741 & 0 & 1 & 1 \\
\hline 060810143004.730 & 0.805664 & 0.650977 & 1.005469 & 3211.158974 & 386 & 1 & 2 & 1 \\
\hline 060810143011.309 & 1.65 & 1.65 & 3.003516 & 139150.2222 & $3,277,056$ & 259 & 2 & 62 \\
\hline 060810143015.085 & 0.805664 & 0.647754 & 1.005469 & 3219.413882 & 387 & 1 & 2 & 1 \\
\hline 060810182951.567 & 0.818555 & 0.673535 & 1.028027 & 3107.573201 & 2095 & 1 & 3 & 1 \\
\hline 060810182959.678 & 1.65 & 1.65 & 2.085059 & 139150.2222 & $4,391,168$ & 259 & 3 & 105 \\
\hline 060810183001.231 & 0.818555 & 0.66709 & 1.028027 & 3107.573201 & 2096 & 1 & 3 & 1 \\
\hline 060810142629.200 & 3.20332 & 3.270996 & 0.944238 & 2619.983264 & 961 & 1 & 4 & 2 \\
\hline 060810142631.833 & 1.65 & 0 & 0.141797 & 208725.3333 & $6,947,328$ & 663 & 4 & 7 \\
\hline 060810142639.434 & 3.20332 & 3.270996 & 0.944238 & 2614.51357 & 962 & 1 & 4 & 2 \\
\hline
\end{tabular}

the line in bold in Table 2 does not explicitly show that data loss occurred at Node 2. Only if one relates to the previous line with Node 2 as parent ID, then one can tell that the sequential number 166 is lost. To generalize this identification procedure to detect all lost data, the formatted data needs to be sorted according to node ID. The lines associated with the same nodes are saved into individual data files. Within each data file, the lines are organized according to time stamp in ascending order, and then sequential numbers are searched. Each missing sequential number is related to one lost data point. In this method, lost data is identified, and the yield at the node can be calculated (see section 'spatial correlation and yield').

Erroneous Data: This problem stands out more peculiarly in the raw data than the data loss problem. Table 3 illustrates out-of-the-range readings in Columns 2-7 and 9, which is an example of the first subtype of erroneous data. This subtype of erroneous data, in essence, contains certain routing information (UC Berkeley, 2004). In future studies, it may be further excluded from the data that is passed onto an end user. In this study, it is discarded in the data cleansing stage because of wrong readings in the aforementioned columns. The second subtype of erroneous data refers to the repetition of the same line of information (except for the time stamp) once or twice in the recorded data as illustrated in Table 4. Note that this subtype of erroneous data is also observed when processing the data collected by using Surge Reliable (Ferzli et al., 2006b, a; Pei et al., 2007). Screening the data according to sequential number can identify this problem. This subtype of erroneous data, in short, should not be used to extract information because it does not contain any new information itself.

The above observations are the foundation of the subsequent data cleansing effort (see section 'data cleansing effort'). Although seemingly sufficient in this study from the viewpoint of an end user of 'Smart Dust' (who would primarily need to process the data and extract relevant information), the nature of the data problems is subjected to further examination. More importantly, these data problems are being verified theoretically based on a thorough understanding of the inner-workings of Mica2 Motes and TinyOS in an 
Table 4. A segment of sorted data for Node 1 to illustrate the second subtype of erroneous data. The data is extracted from the results of a test conducted on August 8, 2006. Lines with the same modification contain repetitive information, except for the time stamp.

\begin{tabular}{|c|c|c|c|c|c|c|c|c|}
\hline $\begin{array}{l}\text { Time stamp } \\
\text { (yymmddHHMMSS.FFF) } \\
\text { (1) }\end{array}$ & $\begin{array}{l}\text { Ice } \\
\text { (volt) } \\
(2)\end{array}$ & $\begin{array}{l}\text { Leaf } \\
\text { (volt) } \\
(3)\end{array}$ & $\begin{array}{c}\text { Temperature } \\
\text { (volt) } \\
\text { (4) }\end{array}$ & $\begin{array}{c}\text { Battery } \\
\text { (millivolt) } \\
(5)\end{array}$ & $\begin{array}{l}\text { Sequential } \\
\text { no. (no unit) } \\
\text { (6) }\end{array}$ & $\begin{array}{l}\text { Parent ID } \\
\text { (no unit) } \\
(7)\end{array}$ & $\begin{array}{l}\text { Node ID } \\
\text { (no unit) } \\
(8)\end{array}$ & $\begin{array}{c}\text { Logic } \\
\text { (no unit) } \\
\text { (9) }\end{array}$ \\
\hline 060809165550.004 & 0.802441 & 0.644531 & 0.999023 & 3130.88 & 1616 & 0 & 1 & 1 \\
\hline 060809165600.799 & 0.802441 & 0.641309 & 0.999023 & 3130.88 & 1617 & 0 & 1 & 1 \\
\hline 060809165601.510 & 0.802441 & 0.641309 & 0.999023 & 3130.88 & 1617 & 0 & 1 & 1 \\
\hline 060809165610.884 & 0.802441 & 0.641309 & 0.999023 & 3130.88 & 1618 & 0 & 1 & 1 \\
\hline 060809165620.778 & 0.802441 & 0.638086 & 0.999023 & 3130.88 & 1619 & 0 & 1 & 1 \\
\hline 060809165629.971 & 0.802441 & 0.631641 & 0.999023 & 3130.88 & 1620 & 0 & 1 & 1 \\
\hline 060809165636.891 & 0.802441 & 0.625195 & 0.995801 & 3130.88 & 1621 & 0 & 1 & 1 \\
\hline 060809165641.708 & 0.802441 & 0.625195 & 0.995801 & 3130.88 & 1621 & 0 & 1 & 1 \\
\hline 060809165646.885 & 0.799219 & 0.580078 & 0.989355 & 3130.88 & 1622 & 0 & 1 & 1 \\
\hline 060809165700.506 & 0.802441 & 0.654199 & 0.999023 & 3130.88 & 1623 & 0 & 1 & 1 \\
\hline 060809165710.420 & 0.802441 & 0.654199 & 0.999023 & 3130.88 & 1624 & 0 & 1 & 1 \\
\hline 060809165720.233 & 0.802441 & 0.654199 & 0.999023 & 3130.88 & 1625 & 0 & 1 & 1 \\
\hline 060809165726.894 & 0.802441 & 0.650977 & 0.999023 & 3130.88 & 1626 & 0 & 1 & 1 \\
\hline 060809165741.545 & 0.802441 & 0.673535 & 1.002246 & 3130.88 & 1627 & 0 & 1 & 1 \\
\hline 060809165750.217 & 0.802441 & 0.650977 & 0.999023 & 3130.88 & 1628 & 0 & 1 & 1 \\
\hline 060809165800.792 & 0.802441 & 0.647754 & 0.999023 & 3138.726817 & 1629 & 0 & 1 & 1 \\
\hline 060809165810.345 & 0.802441 & 0.647754 & 0.999023 & 3130.88 & 1630 & 0 & 1 & 1 \\
\hline 060809165819.849 & 0.802441 & 0.644531 & 0.999023 & 3130.88 & 1631 & 0 & 1 & 1 \\
\hline 060809165831.456 & 0.802441 & 0.676758 & 1.002246 & 3130.88 & 1632 & 0 & 1 & 1 \\
\hline 060809165840.480 & 0.802441 & 0.641309 & 0.999023 & 3130.88 & 1633 & 0 & 1 & 1 \\
\hline 060809165850.473 & 0.802441 & 0.647754 & 0.999023 & 3130.88 & 1634 & 0 & 1 & 1 \\
\hline 060809165900.138 & 0.802441 & 0.638086 & 0.999023 & 3130.88 & 1635 & 0 & 1 & 1 \\
\hline 060809165910.221 & 0.802441 & 0.634863 & 0.999023 & 3130.88 & 1636 & 0 & 1 & 1 \\
\hline 060809165920.467 & 0.802441 & 0.628418 & 0.995801 & 3130.88 & 1637 & 0 & 1 & 1 \\
\hline 060809165931.552 & 0.799219 & 0.580078 & 0.989355 & 3130.88 & 1638 & 0 & 1 & 1 \\
\hline 060809165940.325 & 0.802441 & 0.657422 & 0.999023 & 3130.88 & 1639 & 0 & 1 & 1 \\
\hline 060809165941.967 & $\overline{0.802441}$ & $\overline{0.657422}$ & 0.999023 & 3130.88 & $\overline{1639}$ & $\overline{0}$ & $\overline{1}$ & $\overline{1}$ \\
\hline 060809165942.608 & $\overline{0.802441}$ & 0.657422 & 0.999023 & $\overline{3130.88}$ & $\overline{1639}$ & $\overline{0}$ & $\overline{1}$ & $\overline{1}$ \\
\hline 060809165950.009 & 0.802441 & 0.654199 & 0.999023 & $\overline{3130.88}$ & $\overline{1640}$ & $\overline{0}$ & $\overline{1}$ & $\overline{1}$ \\
\hline
\end{tabular}

ongoing pursuit of the authors and their collaborators. In this study, more questions exist than answers when learning the capabilities of Mica2 Motes, which is documented in Pei et al. (2007). One of the scenarios experienced in this study is highlighted here to indicate the complexity associated with data collected by Mica2 Motes (which necessitates improvements in understanding):

Malfunctioning of battery power reading happens when processing the data at the nodes connected to the SRB and when the reading of battery power starts with a low value. To clearly depict this scenario, Table 5 presents a segment of data that is extracted from the test on September 14, 2006. All the readings seem to be error-free and thus can be utilized in data processing except those readings of battery voltage in Column 5 that are highlighted in bold. Note that this does not fall under the first subtype of erroneous data. The battery reading was already as low as $2450.786693 \mathrm{mv}$ when the eccentric reading came into the picture. There are also data losses during the gap of the data, i.e., between 5:10:19 $\mathrm{pm}$ and 7:08:13 $\mathrm{pm}$ on that day of testing. This was conveniently identified according to the discontinuous sequential numbers. The lines associated with eccentric readings in the battery might not be considered as erroneous lines. This is because the information contained in Columns $2-4,6-7$, and 9 can be trusted and utilized in the subsequent data analysis.

\section{Data Cleansing Effort}

To address the aforementioned data problems, a data cleansing effort is carried out. This lays a foundation for the subsequent analysis of the network performance as well as the performance of the developed Sensor-Road Button (SRB) that is connected to the wireless network.

For lost data, quantitative measures can be conveniently associated with a sequential number rather than a time stamp. Lost data, as experienced in this study, can be effectively identified by tracking all sequential numbers at each node (rather than at Node 0 alone; i.e., Nodes 1-4 in this study) and making a reference to all sequential numbers at Node 0 . Note that (i) the sequential numbers at each node would be perfectly consecutive if there were never any lost data, and (ii) the sequential 
Table 5. One segment of sorted data for Node 1 to illustrate a special erroneous data problem (in bold) caused by low battery voltage. The data is extracted from the results of a test conducted on September 14, 2006. Note that the embedded algorithm worked properly at that moment, thus correct logic results were shown at Node 1 when dry ice was deposited to mimic an icy condition.

\begin{tabular}{|c|c|c|c|c|c|c|c|c|}
\hline $\begin{array}{l}\text { Time stamp } \\
\text { (yymmddHHMMSS.FFF) } \\
\text { (1) }\end{array}$ & $\begin{array}{l}\text { Ice } \\
\text { (volt) } \\
(2)\end{array}$ & $\begin{array}{l}\text { Leaf } \\
\text { (volt) } \\
(3)\end{array}$ & $\begin{array}{c}\text { Temperature } \\
\text { (volt) } \\
\text { (4) }\end{array}$ & $\begin{array}{c}\text { Battery } \\
\text { (millivolt) } \\
(5)\end{array}$ & $\begin{array}{l}\text { Sequential } \\
\text { no. (no unit) } \\
\text { (6) }\end{array}$ & $\begin{array}{l}\text { Parent ID } \\
\text { (no unit) } \\
(7)\end{array}$ & $\begin{array}{l}\text { Node ID } \\
\text { (no unit) } \\
(8)\end{array}$ & $\begin{array}{c}\text { Logic } \\
\text { (no unit) } \\
\text { (9) }\end{array}$ \\
\hline 060914170919.043 & 2.987402 & 3.206543 & 1.401855 & 2450.786693 & 1898 & 0 & 1 & 0 \\
\hline 060914170929.178 & 2.98418 & 3.20332 & 1.401855 & Inf & 1899 & 0 & 1 & 0 \\
\hline 060914170936.388 & 2.98418 & 3.19043 & 1.401855 & 2450.786693 & 1900 & 0 & 1 & 0 \\
\hline 060914170949.457 & 2.98418 & 3.19043 & 1.401855 & 2450.786693 & 1901 & 0 & 1 & 0 \\
\hline 060914170959.482 & 2.98418 & 3.183984 & 1.405078 & 2450.786693 & 1902 & 0 & 1 & 0 \\
\hline 060914171019.020 & 2.980957 & 3.035742 & 1.408301 & 2450.786693 & 1904 & 0 & 1 & 0 \\
\hline 060914190813.013 & 2.829492 & 3.129199 & 2.645801 & $1,252,352$ & 2612 & 0 & 1 & 2 \\
\hline 060914190823.276 & 2.829492 & 3.129199 & 2.645801 & $1,252,352$ & 2613 & 0 & 1 & 2 \\
\hline 060914190833.711 & 2.803711 & 3.106641 & 2.626465 & $1,252,352$ & 2614 & 0 & 1 & 2 \\
\hline 060914190843.756 & 2.797266 & 3.106641 & 2.62002 & $1,252,352$ & 2615 & 0 & 1 & 2 \\
\hline 060914190853.750 & 2.797266 & 3.106641 & 2.623242 & $1,252,352$ & 2616 & 0 & 1 & 2 \\
\hline 060914190903.384 & 2.797266 & 3.106641 & 2.623242 & 62,6176 & 2617 & 0 & 1 & 2 \\
\hline 060914190913.138 & 2.797266 & 3.103418 & 2.623242 & $1,252,352$ & 2618 & 0 & 1 & 2 \\
\hline 060914190923.543 & 2.794043 & 3.100195 & 2.62002 & $1,252,352$ & 2619 & 0 & 1 & 2 \\
\hline 060914190933.537 & 2.79082 & 3.096973 & 2.616797 & Inf & 2620 & 0 & 1 & 2 \\
\hline 060914190943.351 & 2.794043 & 3.100195 & 2.62002 & $1,252,352$ & 2621 & 0 & 1 & 2 \\
\hline
\end{tabular}

numbers at each nonzero node would be identical to those at Node 0 if there were zero initial connection time and zero time lag in the transmission of signal.

For erroneous data, each line in a formatted data file should be searched through, as can be seen from the examples presented in Section 'Typical data problems associated with Surge Time Synchronization'. The errors identified in this process would then be conveniently presented in a plot of the error occurrence versus time stamp, which would possess a more transparent meaning (i.e., time histories) than that seen when using the associated sequential number. The first subtype of erroneous data can be identified based on the peculiar features that it possesses in these specified columns. The other subtype of erroneous data can be identified by comparing it with its neighboring data.

These relatively straightforward arguments form the basis for the automation of the data cleansing procedure (Pei et al., 2007). As an intermediate step, a history of packet/data reception versus sequential number is plotted for each node in each test. A sample plot is presented in Figure 6, where a received and lost packet/ data point is represented by one and zero, respectively.

\section{Spatial Correlation and Yield}

Following Figure 6, for each node in each test, there is a binary vector $x$ with the length of the total number of sequential numbers sent from Node $0, n$. When a packet is received, the corresponding values for $x$ equal one; conversely, if a packet is not received, the corresponding values for $x$ are set to zero. Using these vectors, spatial correlation of data loss can be calculated following Zhao and Govindan (2003). Between two distinct nodes, excluding Node 0 , cross-correlation coefficients can be calculated and used as a measure for spatial correlation of packet delivery performance, in this context, data delivery performance. The cross-correlation coefficient is defined below (following Zhao and Govindan, 2003):

$$
R_{i, j}=\frac{\sum_{k=1}^{n} x_{i k} x_{j k}-n \bar{x}_{i} \bar{x}_{j}}{\sqrt{\left[\sum_{k=1}^{n} x_{i k}^{2}-n \bar{x}_{i}^{2}\right]\left[\sum_{k=1}^{n} x_{j k}^{2}-n \bar{x}_{j}^{2}\right]}}
$$

where $i$ and $j$ denote the node IDs, and $i \neq j$. The vectors of $x$ 's are explained as above. Also, $\bar{x}_{i}$ and $\bar{x}_{j}$ are mean values of the series of $x_{i}$ and $x_{j}$, respectively (following the commonly used notation).

Choices of proper quantities such as reception rate and required number of packets used to describe the quality of wireless links are discussed in Cerpa et al. (2005). In this study, yield is utilized for practical purposes. When a valid packet is received from the node at a specified time stamp, the yield of the specified node is the ratio between the number of actually received packets and the number of possibly received packets of the specified node. The yield at each node, which is a function of time stamp rather than sequential number, should be consistent with the history of data reception rather than sequential number. In this study, the yield is calculated according to the following formula:

$$
\text { Yield }=\frac{\text { Number of packets that have been received by the node }}{\text { Current sequential number at the given node }- \text { the number of the initial sequential number at Node } 0}
$$



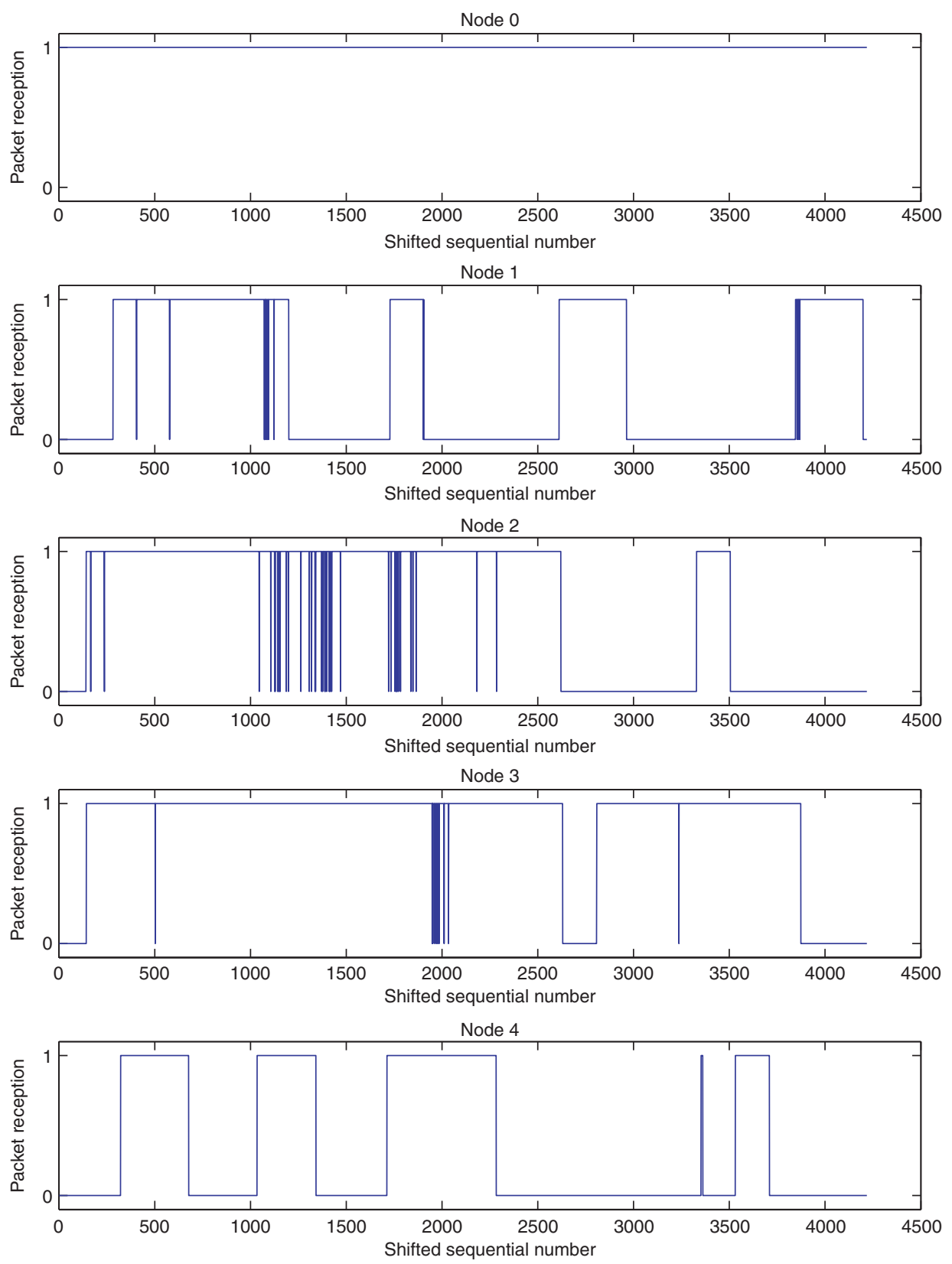

Figure 6. Packet/Data reception versus sequential number for each node following the test result on September 14, 2006. Note that one and zero represent a received and lost packet/data point, respectively.

For several typical (as well as idealized) scenarios, the values of yield are discussed as follows:

- If a node is never connected to the network, then its yield is not defined throughout the testing time. This shows the influence of the connectivity to the defined yield.

- If a node becomes disconnected from the network after a certain time stamp, then its yield is no longer defined. This, again, shows the influence of the connectivity to the defined yield. In other words, the defined yield can be used to reflect the connectivity of the network.
- If a node becomes connected to the network at a certain time stamp, then its yield becomes defined (beginning at that point) and should show an increasing trend starting at zero. This shows the influence of the initial connection time of the node to the defined yield.

- If there is no data loss throughout the testing time and the node stays connected to the network after the initial connection time, then the yield will start at zero, begin increasing, and asymptotically approach one.

- If there is a period of time with data loss at the specified node, then the yield is not defined during 
that period of time. Furthermore, there is a downward trend in the yield during the 'gap' in time. This downward trend is an indicator of data loss.

- Ideally, the yield can be $100 \%$ throughout the testing time if there is no discontinuity or data loss, and the initial connection time is zero.

A limitation in this definition of yield is that it is incomplete; it is undefined when there is a discontinuity between the specific node and the network. One could estimate the number of possibly received packets in the denominator of the expression in Equation (2) by utilizing the known sampling rate. This alternative, however, is not preferred in this study since irregularities in the recorded sampling intervals have been observed in the collected data (Pei et al., 2007). This further justifies the definition of the yield in Equation (2). In summary, the yield defined in Equation (2) is an accumulated measure for packet reception (once again, data reception in this context), and as discussed above, it can be used to reflect the connectivity, initial connection time, and data loss of the specified node.

\section{RESULTS AND ANALYSIS}

\section{Practical Concerns on Performance of Wireless Sensor Network}

The guiding interest of this study is to evaluate the performance of Mica2 Motes by properly designing tests and thoroughly analyzing data. Practical concerns regarding the implementation of an off-the-shelf wireless sensor product like Mica2 Motes in the specified application would include (i) connectivity, (ii) transmitting range, (iii) initial connection time, (iv) data reception rate and erroneous data, (v) flexibility and robustness in the real-world, e.g., the acclaimed 'selfhealing' capability, (vi) performance at irregular intervals or alignments, and (vii) battery life.

As outlined in the section 'Data cleansing and processing', lost and erroneous data is first attended to by a data cleansing effort. The yield of each node and the spatial correlation of the nodes are then calculated. Data collected from each test at Stages III and IV is processed and presented in a format as in Figures 7 and 8 . Using this format, the space designated to a node will be intentionally left blank whenever this node was not connected in a test. Note that these two figures are selected as examples since all of the motes were connected in these tests showing the best performance and great potential for a Mica2 Mote sensor network in addition to their not having wasted space. The not-so-successful tests are summarized and shown in Figure 9.
The design of both Figures 7 and 8 are further explained as follows:

- Pictures are included to illustrate the adopted testing configuration including the layout (especially the location of Node 0$)$, interval $(25,50$, or 100 feet), height (following which type of the posts is used in the test) and packaging condition (with or without the antenna plates and bags for waterproofing) of the motes.

- Curves are drawn to visualize the spatial correlation between the nodes for the entire duration of the test following Equation (1).

- Time history plots occupying the remainder of the figure include the yield calculated according to Equation (2), the occurrence frequency of the first (blue crosses) and second (red circles) subtypes of erroneous data as presented in section 'typical data problems associated with surge time synchronization', the parent relationship, and the battery voltage.

Having all individual results as in Figures 7 and 8 is helpful to appreciate the versatile performance of Mica2 Motes as there are a wide range of varieties of the performance (Pei at al., 2007). The performance of motes has been reported to vary drastically (e.g., in terms of packet loss as in Zhao and Govindan (2003); thus, this versatility may or may not be entirely caused by the introduced variations in mote layout, interval, height, and the condition of dipole effect. In other words, examining each realization in the random processes of the performance of the selected wireless sensor network may not be sufficient. Statistical measures of these realizations are needed to achieve a good understanding of the overall performance of Mica2 Mote wireless sensor networks to more directly address these practical concerns.

\section{Performance of Mica2 Motes}

Figure 9 is designed to show both connectivity and transmitting range. The reliability of wireless technologies is quite challenging especially for a low-cost technical solution adopted in this study. The environmental factors are known to affect the performance of wireless channels (e.g., Shankar, 2002; Mark and Zhuang, 2003). In addition, extreme temperatures including both high and low are adverse to the performance of electronic devices in general. Figure 9 is designed to summarize what has been learned from the experimental investigation in this study, showing the connectivity of Mica2 Motes as dependant on (i) the transmitting range, (ii) the height of the motes, and (iii) the possible correlation with environmental temperature. 

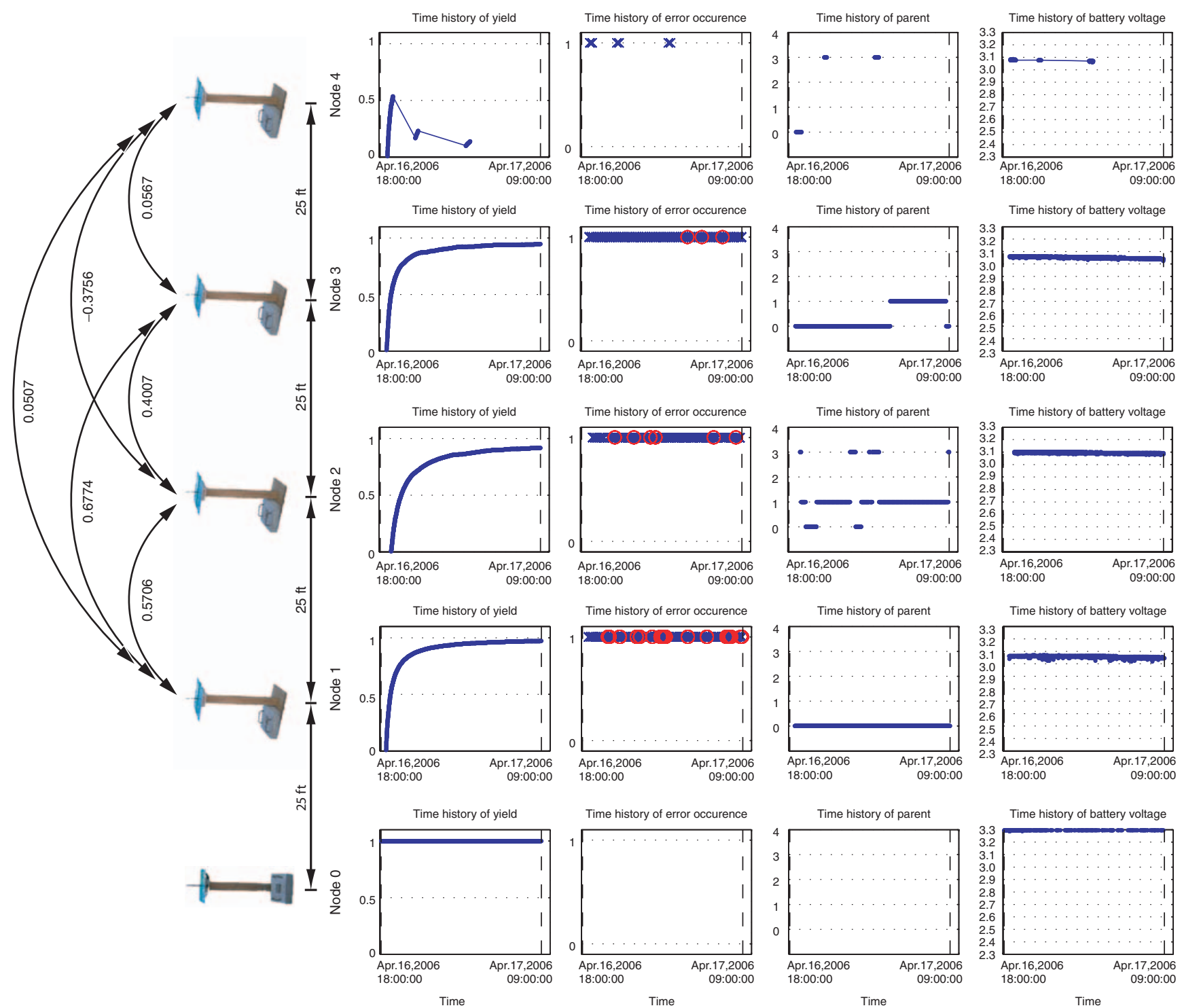

Time history of error occurence
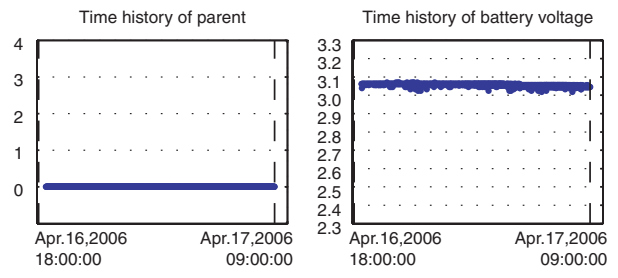

Figure 7. Testing configuration and collected time histories starting on April 16, 2006. In each panel of time histories, two dashed lines in black are drawn to indicate the starting and ending points of the test according to Node 0 . The occurrence frequencies of the first (with peculiar values) and second (with repetitive information) subtypes of erroneous data, as discussed in the section 'Typical data problems associated with surge time synchronization', are shown in blue crosses and red circles, respectively.

Given the common features of the tests at Stages III and IV presented earlier, their results regarding connectivity are combined into Figure 9. The figure can be considered spatially displaying temporally distributed tests, which will be explained shortly. Figure 9(a) first presents temperature variation during the specified period of the time. Highest and lowest temperatures in Fahrenheit for each day are obtained from Weather Channel (2006). Temperature is selected here as it is an environmental factor that can be easily quantified and has also fluctuated quite substantially during the long testing period. Figure 9(b) is in essence a 3D plot. The $x$-axis indicates the time, while the $y$-axis illustrates all spatial layouts of the Mica2 Motes nodes within the period of the time. The result of each test takes up one vertical line in the plot, while the concatenation of these vertical lines along the $x$-axis unfolds all the test results. A table would be adopted for this presentation instead of using this figure, however, plotting these results into one figure can better illustrate the spatial relationship of nodes from test to test.

As shown in Figure 9, the connectivity at Stage III seems to be better than that at Stage IV. First of all, shorter mote intervals were adopted at Stage III. Posts with different heights were used in different tests including 33 inches, 6 and 9 feet and on two occasions, the motes were put on the ground. It can been seen that most of the tests in the yard (with both 25 and 50 feet as mote intervals) had good overall connectivity, however the tests along the fence did not. The tests at Stage IV do 


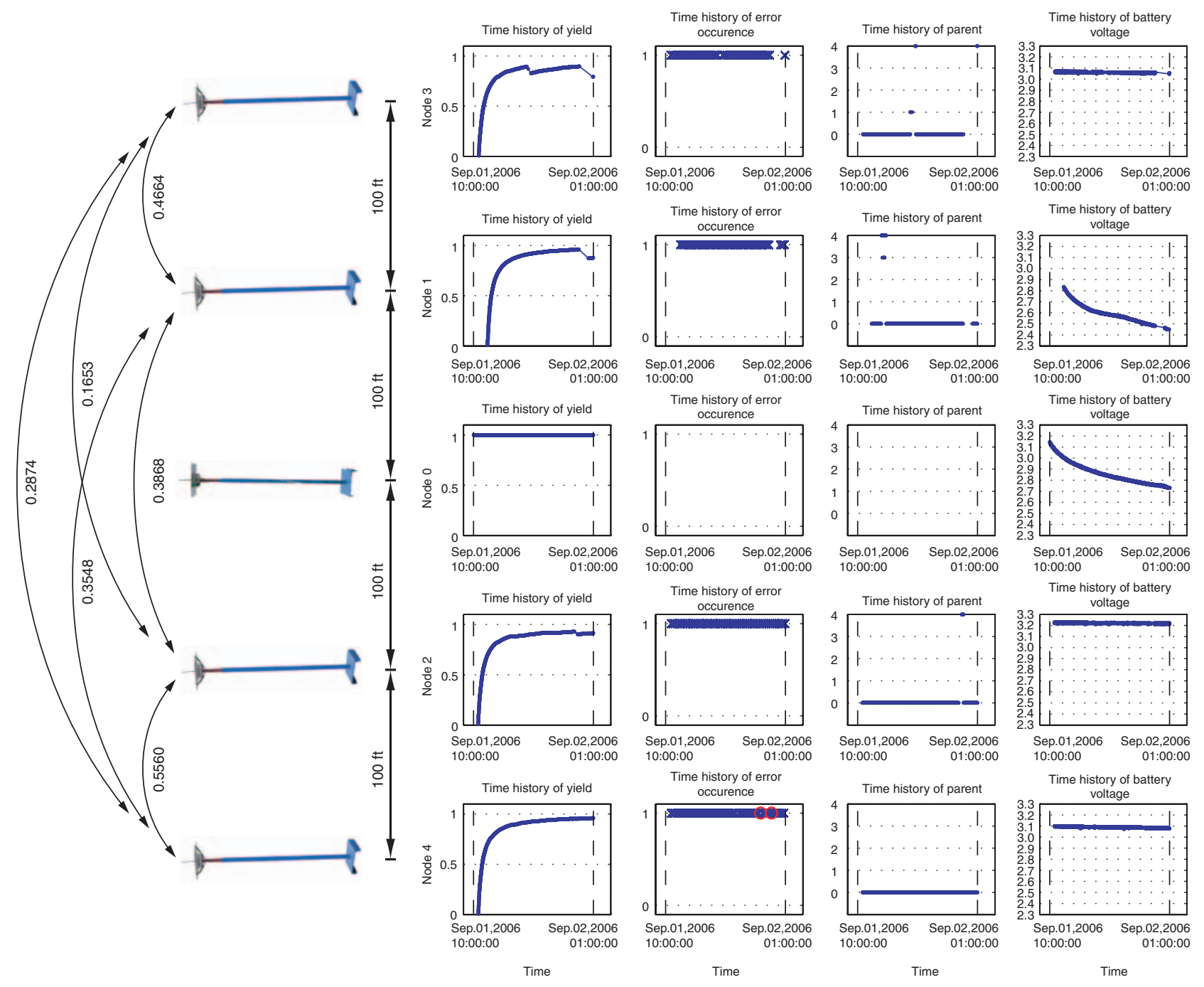

Figure 8. Testing configuration and collected time histories starting on September 1, 2006. In each panel of time histories, two dashed lines in black are drawn to indicate the starting and ending points of the test according to Node 0 . The occurrence frequencies of the first (with peculiar values) and second (with repetitive information) subtypes of erroneous data, as discussed in the Section 'Typical data problems associated with surge time synchronization', are shown in blue crosses and red circles, respectively.

not have good connectivity when the short timber posts were adopted and the temperature appeared to be high. The physical strain of the hardware components, especially the RF connectors, might contribute to the poor performance of the connectivity at mid-Stage IV as revealed by the trouble shooting effort reported in Pei et al. (2007).

Figure 10 presents an assembly of packet reception rates, which complement packet loss rates. Packet loss is commonly used to measure the reliability of the network connectivity, as is packet reception. As presented previously in the section 'Format of data collected by mica2 motes using surge time synchronization', one line in the collected formatted data file can be considered one packet, thus data losses can be conveniently associated with missing sequential numbers. Packet reception rate at every node is calculated as the ratio of the total number of received packets at the specified node over the total number of packets sent by Node 0 . Packet reception rate at Node 0 remains $100 \%$ since it was always wired to the laptop in the study. Most of the tests shown in Figure 9 are used to plot Figure 10, except for the tests conducted at early Stage IV (July 17 to 30, 2006). This is mainly because of the interruptions in the data caused by the resetting of motes during the testing on those trial days for the road tests.

Packet reception rate is plotted as a function of the location of the nodes in each panel in Figure 10. Within each panel, large variations in packet reception rate can be observed from test to test. For a specific node, there can be a large dispersion in packet reception such as that at Location $Y \# 25$ in Figure 10(a), Locations $Y \# 125, \quad Y \# 100$ and $Y \# 50$ in Figure 10(b), Locations $Y \# 175, Y \# 125$ and $Y \# 25$ in Figure 10(c), Location $F \# 50$ in Figure 10(d), Locations $R \# 0, R \# 100$, $R \# 300$ and $R \# 400$ in Figure 10(e), and Locations $R \# 100$ 
(a)
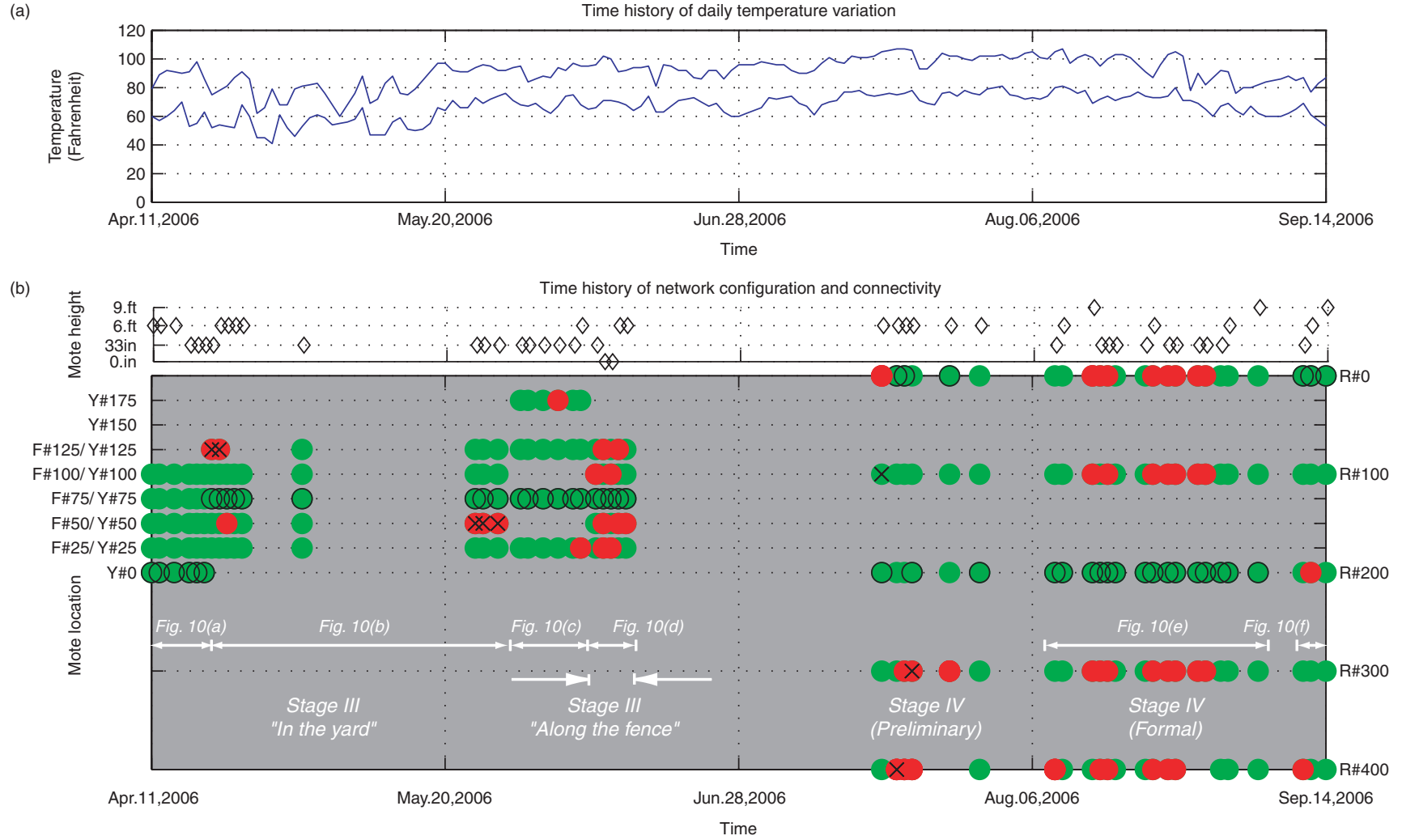

Figure 9. Overview of network connectivity for almost all successfully recorded tests in Stage III, the 'overnight tests', and Stage IV, the 'road tests', and possible correlation between daily temperature variation and the network connectivity during the same period of testing. The highest and lowest daily temperatures are depicted in Part (a), where the data is obtained from Wea (2006). In Part (b), the mote interval of each Mica2 Motes sensor network is drawn to scale. The color code used in this figure is as follows: Green and red dots depict connected and nonconnected nodes, respectively. Green dots with black rims represent the locations of Node 0's, while those nodes suspected of having a hardware problem are marked with a cross. The data used for each panel in Figure 10 is also indicated.

and $R \# 400$ in Figure 10(f). In contrast, there are some nodes of which the packet reception can be quite consistent such as those at Locations $Y \# 100, Y \# 75$ and $Y \# 50$ in Figure 10(a), Location $Y \# 25$ in Figure 10(b), Locations $F \# 125, F \# 100$ and $F \# 25$ in Figure 10(d), and Locations $R \# 200$ and $R \# 300$ in Figure 10(f). Regardless of these fluctuations, there is an overall trend for the packet reception rate to decrease with the transmitting range. This inversely proportional relationship is consistent with commonly seen theoretical or empirical models (e.g., Shankar, 2002; Mark and Zhuang, 2003) that are used to predict path loss, i.e., the loss of transmitted energy that is closely related to packet loss.

Three transmitting environments associated with the results in Figure 10 (including ' $\mathrm{Y}$ ' inside the yard, ' $\mathrm{F}$ ' along the fence, and ' $R$ ' on the road) bear some similarities with each other (Figure 4); however, the differences in these environments may or may not contribute to the differences in the performances of the Mica2 Motes sensor network. Thus, it is chosen not to directly compare the results in Figure 10(a) with those in Figure 10(f), nor those in Figure 10(b) with those in Figure 10(d), and those in Figure 10(e). Nevertheless, the results in Figure 10(b) can be compared with those in Figure 10(c) on a light-to-light basis. It seems that switching from a shorter to a longer mote interval (i.e., 25 to 50 feet) does not necessarily decrease the packet reception rate of the wireless sensor network. In this study, the mote interval of the selected Mica2 Motes is confidently stretched to 100 feet without compromising the performance. This shows the promise for 'Smart Dust' to be applied in large-scale systems, the topic of which has not been sufficiently explored in previous studies (see section 'Introduction').

The initial connection time spent by each mote in all tests can be evaluated by using a figure similar to Figure 10 but in semi-log plots. The default initial connection time of 30 min was adopted in this study. The variation in initial connection time is found to be quite large, from a minimum of 24.293 to a maximum of 522.379 with an average value of 54.201 - all in minutes. Also, it seems that a node that is placed further away from Node 0 may not take more time to connect than one that is closer to Node 0 . This may be a result of the ad hoc nature of the adopted Mica2 Motes sensor network.

The statistics of the erroneous data as defined in the section 'Typical data problems associated with surge 
time synchronization' are analyzed in Pei et al. (2007). Error rate at every node is calculated as the ratio of the number of prescribed error(s) at the specified node to the total number of packets sent by the node. The percentage of the occurrence of total errors is fairly low, which is less than 3\% (6\% for the 'road tests' when Node 0 was placed in the middle of the chain).
These values are lower than the error rate of $8 \%$ cited in Koushanfar and Potkonjak (2005).

Even though a direct and explicit explanation of the 'self-healing' features of Mica2 Motes is not available in this study, some snapshots can be provided to understand the ad hoc nature of Mica2 Motes wireless sensor network. The third column of time history plots

(a)

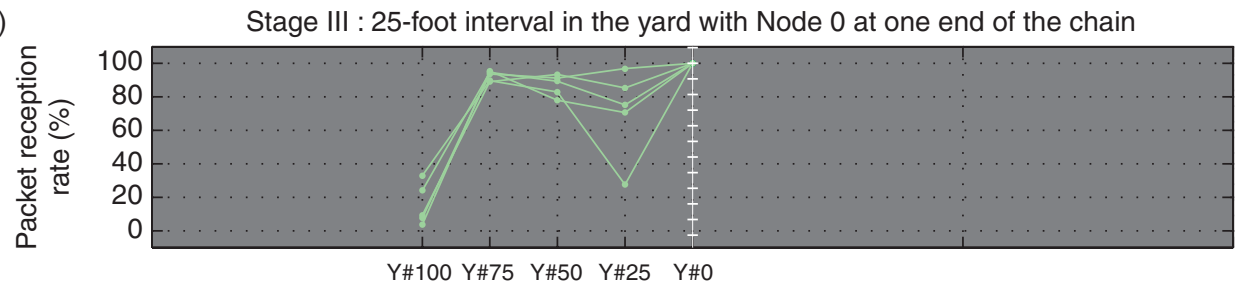

(b)

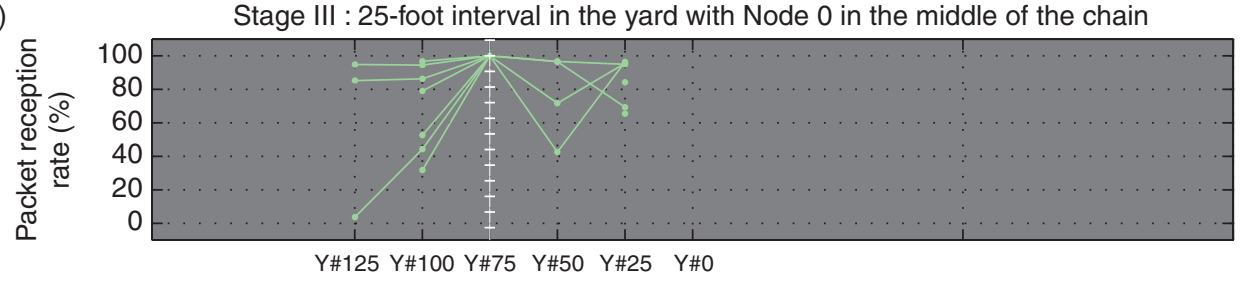

(c)

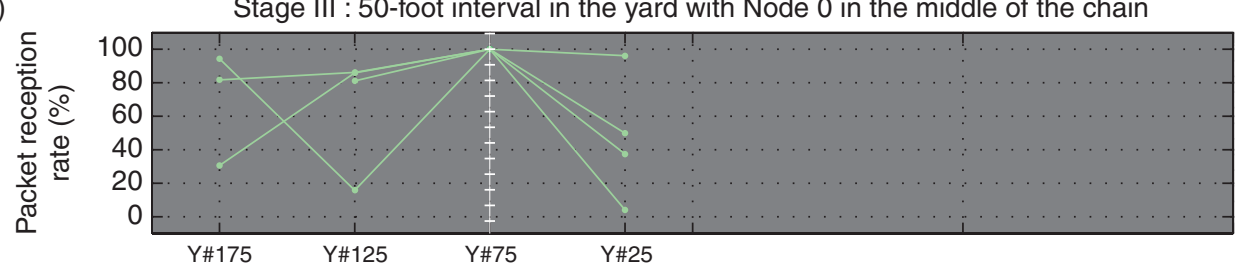

(d)

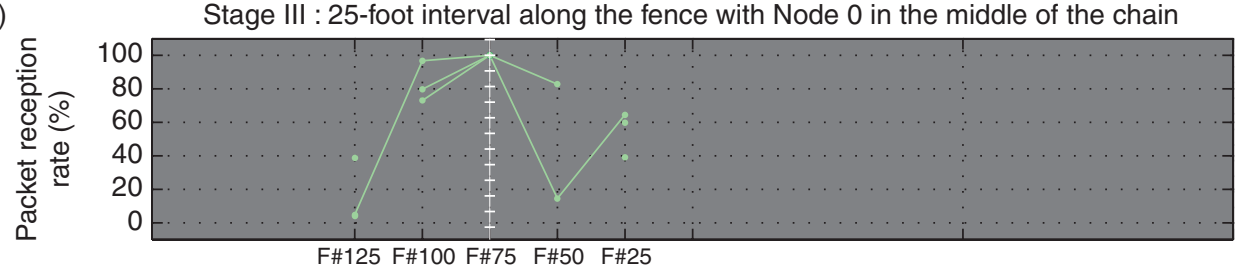

(e)

Stage IV : 100 -foot interval on the road with Node 0 in the middle of the chain

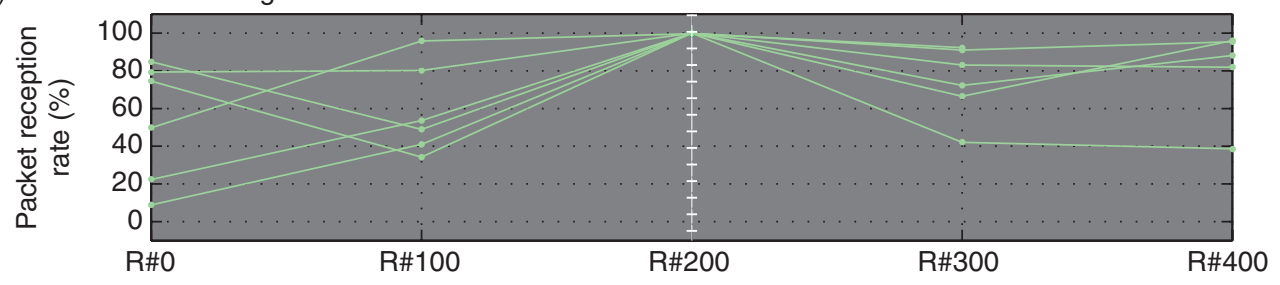

(f)

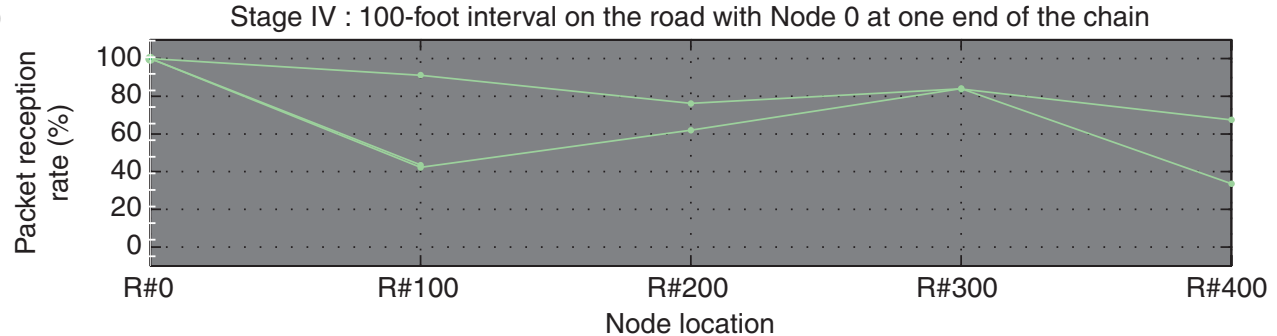

Figure 10. Summary of packet reception rates in most of tests conducted in Stages III and IV. Refer to Figure 4 for the location IDs. White broken lines indicate the location of Node 0 . Points are connected to their adjacent points in the same test. Discrete points are caused by disconnections of the adjacent nodes in the same test. 
in Figures 7 and 8, for example, reveals an interesting facet of the adopted ad hoc wireless sensor network. Other parents rather than Node 0 can be seen for Nodes 1 to 4 . Differing tremendously from the intuitive but wrong perception at Stage II of this experimental study (Ferzli et al., 2006b,a), the network routing feature is very versatile where the motes do not always parent other motes that are located closer to the base station than itself.

\section{Battery Life}

Power consumption (or, battery life when batteries are used) is a major issue to be considered for longterm monitoring. The variation of battery voltage over testing period is examined for this purpose. Battery voltage was tracked from test to test when the same motes were used for the same Node IDs in these tests. For the same time window spanning 16 days and one of these 16 days, respectively, Figure 11 compares the voltages of AA batteries that were used to power the motes during both Stage III, the 'overnight tests,' and Stage IV, the 'road tests.' Note that Node 0 was entirely powered by a wall socket when all overnight tests were conducted (thus its voltage was consistent and did not need to be examined), while it was powered by two AA batteries for all 'road tests.'

As shown in Figure 11(a) and (c), battery voltages decreased gradually in 'overnight' testing when Surge Time Synchronization was applied with no external

(a)
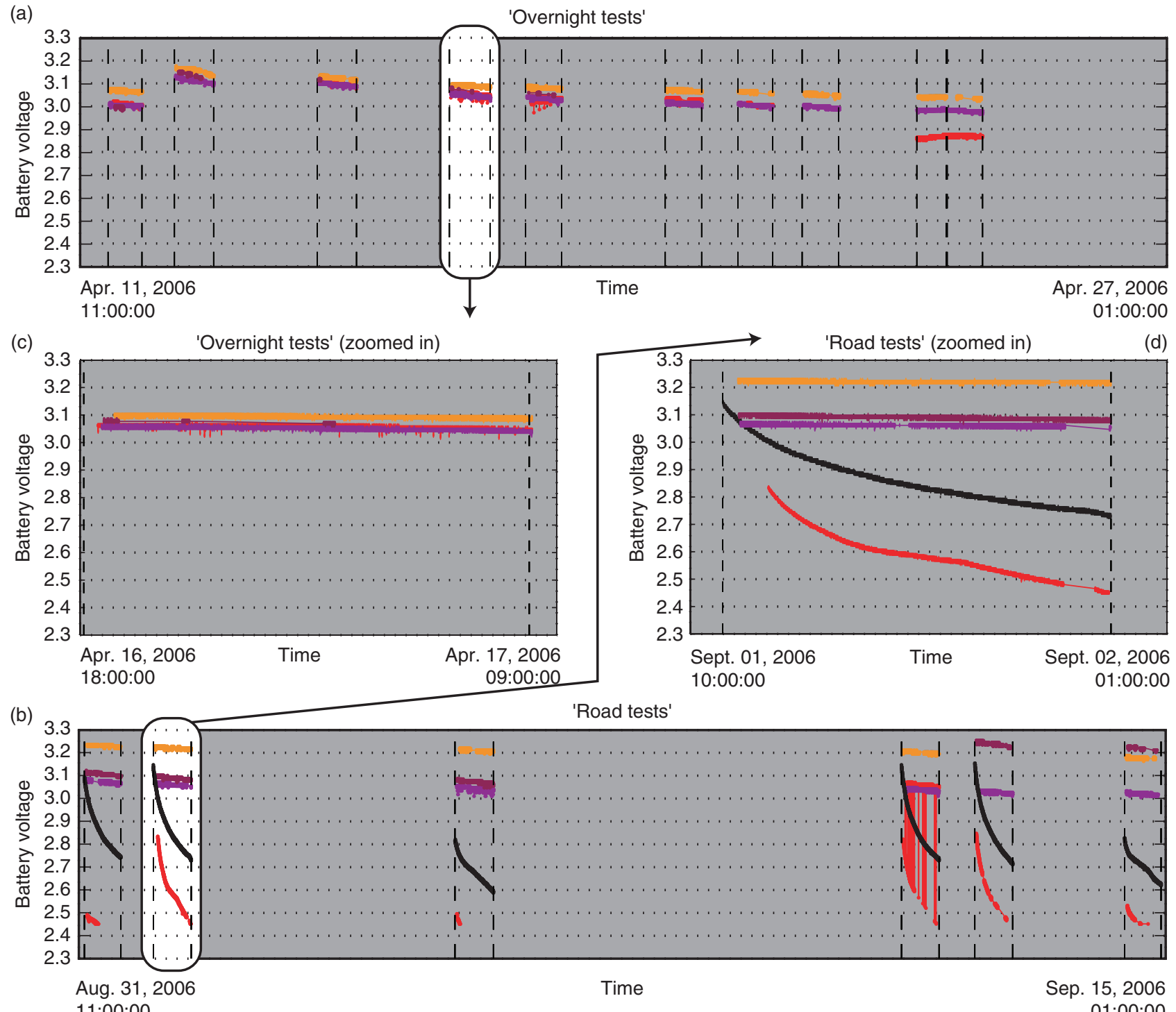

'Road tests'

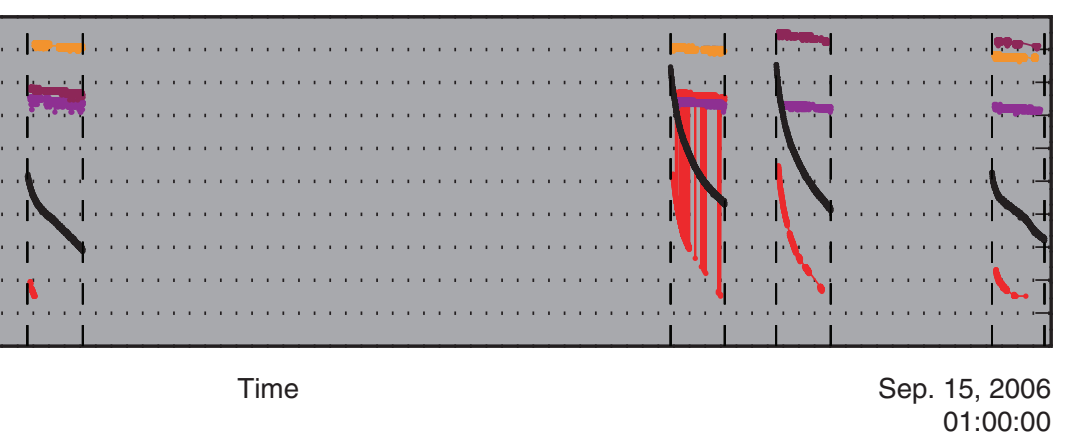

Figure 11. Time histories of battery voltages of all motes during 16 days at (a) Stage III, and (b) Stage IV and those during 15 hours at (c) Stage III, and (d) Stage IV. The color code used in this figure is: Red, orange, purple, and crimson are used to represent the voltage readings from Nodes 1, 2, 3 and 4, respectively. Node 1 was connected to an SRB with three external sensors at Stage IV. Black is used to represent the voltage reading of Node 0 for the 'road tests' only since Node 0 was powered by a wall socket at Stage III. In each time history, two dashed lines in black are drawn to indicate the starting and ending points of the test according to Node 0. 
sensors interfaced with the Motes. Technically, these ordinary AA batteries did not need to be changed every time when about 4320 data points were sampled per test. If the batteries are expected to function properly until they reach approximately $2.7 \mathrm{~V}$ (Crossbow Technologies, 2004), then they can be used for many tests according to the trend shown in both figures. In the results of 'road tests' shown in Figure 11(b) and (d), Nodes 2, 3, and 4 show the same feature of those in the 'overnight' testing, however, both Nodes 0 (the base station) and 1 (which was connected to the SRB housing three external sensors) indicate rapidly decreasing battery voltage in every test. The voltage of Node 1 (in red) on September 11, 2006, fluctuates drastically, which might be caused by an improper connection between the SRB and the mote at Node 1. The phenomenon needs to be further studied for future real-world implementation of the SRB.

\section{Performance of Sensor-Road Button}

The proper functioning of the SRB and some challenges associated with it are depicted in Figure 12. These results are extracted from the data collected at the node that was connected to the SRB. Note that while the voltage outputs of both the leaf wetness and infrared ice sensors are directly presented in the figures, the voltage output of the selected thermistor is converted into the temperature reading in degrees Fahrenheit and plotted in these figures based on the calibration relationship derived from a freeze-thaw chamber test detailed in Pei et al. (2007) and Ferzli et al. (2006b, a).

As detailed in Pei et al. (2007), the raw readings collected by the SRB indicate how the temperature and moisture conditions varied during the testing day. Changes in the thermistor readings are as expected with high readings during the day and low in the night; extremely high temperature readings such as $100-140^{\circ} \mathrm{F}$ can be explained by the high temperature on the road surface and its surrounding air resulting from high atmospheric temperature on those summer days with above $100^{\circ} \mathrm{F}$ reported air temperatures. The readings of the leaf wetness sensor were high throughout since the surface condition of the SRB remained dry. The infrared ice sensor detects the object by emitting an invisible light and then receiving a reflective light. Its performance was found to be quite sensitive. For example, the light from the sun could turn the sensor on, and the sensor could then send the wrong information to the system. This might explain why the voltage output of the sensor was found to be consistently high during daytime and low during the night even though the condition of the SRB remained ice-free on those testing days.

The developed SRB and detection algorithm both seem to have worked properly when the SRB was (i) left in a natural surface condition which was dry (see the first couple of hours in the time histories shown in Figure 12), and (ii) subjected to artificially created rain and icy conditions (on the SRB itself) (see the difference in the results in the time histories shown in Figure 12). When a drop of tap water was dropped onto the surface of the leaf wetness sensor mounted on the SRB, the reading of the sensor and the logic result sent by the node connected to the SRB immediately changed. Figure 12(a) vividly demonstrates the evaporation process of water from the leaf wetness sensor on the SRB. Furthermore, at the end of the test, there was some precipitation, thus some fluctuations in the collected raw readings from the sensors can be seen. Figure 12(b) shows the results from using a bag of dry ice to simulate icy conditions. The moment when dry ice was applied is not captured by the readings on both tests due to data loss. Nevertheless, raw readings of the sensors (especially the thermistor and the infrared ice sensor) as well as the logic result clearly demonstrate the proper functioning of the developed SRB and embedded algorithm under the simulated icy condition.

\section{DISCUSSIONS}

Many limitations in this proof-of-concept study should be noted; all of which may be explored in future studies.

The algorithm illustrated in Figure 1(a) is a quite simple algorithm that fits well within the limited capability of onboard processing power of motes. This algorithm, in any sense, is not an ultimate algorithm to detect road conditions based on a range of measurements that can be achieved by using Mica2 Motes and some low-cost and low-power external sensors. Possible improvements can be made in the following aspects:

Balance the inclusion of raw data and logic results in transmitted data: In this study, both raw data and logic results are included in every transmitted data point. This design offers the convenience to validate the developed system, however, it is not practical. Processing the data and transmitting the processed result would be adopted in a more developed product.

Make the algorithm more robust: The proposed embedded algorithm is an idealized algorithm. First, the dynamic process of ice forming is not considered, i.e., road conditions in the immediate past are not considered. In other words, the prior existence of moisture on the road surface would be considered as an input in this ice detection algorithm in the future. Second, the complications of the selection of decision boundaries in this pattern classification problem are largely simplified as the 

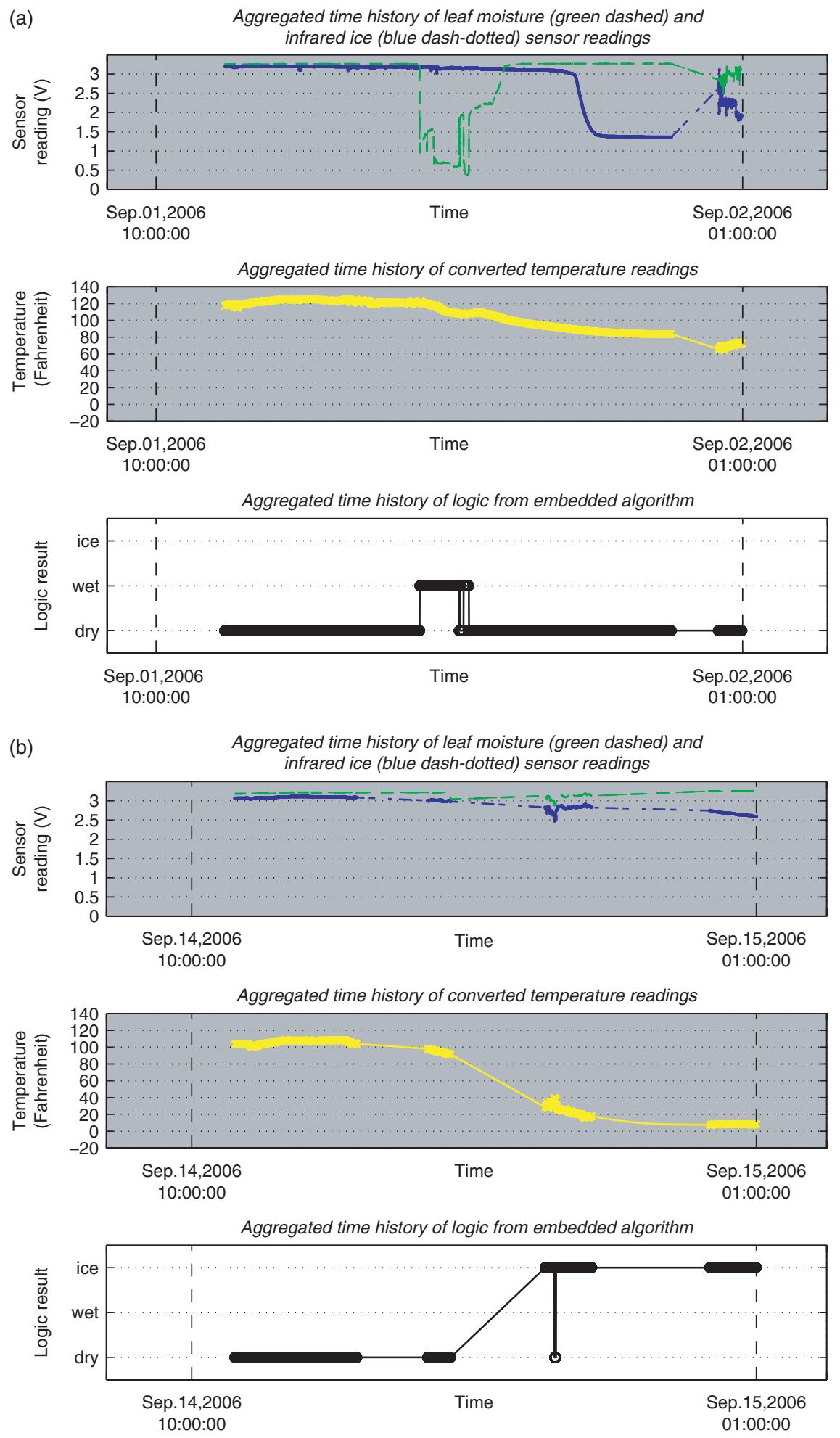

Figure 12. Sensor readings and logic results of Node 1 that was connected to the SRB from tests conducted on (a) September 1, 2006, and (b) September 14, 2006. The yellow, green dashed, and blue dash-dotted lines are for the readings from the thermistor, leaf wetness sensor, and infrared ice sensor, respectively. Water and dry ice were used to simulate rain and icy conditions on the SRB on September 1 and 14, 2006, respectively.

first step to embed more complex algorithms into motes. Last, the noise in the measurements is ignored. This limitation, however, may not affect the functionality of the developed algorithm and monitoring system very much. This is because the detection task in this study is not as complex as in other situations required for monitoring of infrastructure systems and other intelligent transportation related issues. 
Improve the applicable range of the algorithm: It would be worthwhile to consider a wider range of pavement conditions into the embedded algorithm, e.g., frost, and some transitional conditions, such as partially iced and partially wet conditions. The complexity of the algorithm is bounded by the capacity of Mica2 Motes hardware, however this capacity should be exploited and the algorithm could be optimized.

The developed mote packaging method should be further improved to form a truly air-tight casing or evolve into other designs for robust leakage prevention to directly reduce the number of damaged motes in real-world implementation. In addition, the final product of the SRB is still considered as an academic unit by all means. Issues for future improvements include but are not limited to the following: (i) Does the SRB really offer the right measurements that are needed for road condition monitoring? This limitation results from the placement of the SRB as discussed in detail in Pei et al. (2007), (ii) analytical, numerical and experimental studies are needed to examine the survivability of the SRB under accidentally introduced traffic loads, (iii) the effect of roadway debris on the SRB needs to be studied, and the design of the SRB needs to be improved in this regard.

To better validate the developed system for longterm monitoring, a series of long-term uninterrupted tests using the motes will be carried out. In particular, (i) the acclaimed self-healing feature needs to be explicitly validated experimentally preferably in a wireless sensor network of a larger size. (ii) The usefulness of the developed 'Sensor-Road Button' (SRB) needs to be further validated in realworld rainy and icy road conditions. (iii) The newer versions of TinyOS and Surge Time Synchronization could be further explored to possibly improve the relatively short battery life. The 'miniaturization' and integration of the selected external sensors to the motes could also further increase the battery life. (iv) With equal importance, continuous effort should be made towards a better understanding of the inner workings of 'Smart Dust' for better programming of motes and better solutions in data cleansing and data processing.

\section{CONCLUSIONS}

This article presents an effort to bridge the gap between an available technology and a need in practice, i.e., to apply a Mica2 Motes wireless sensor network to detect road surface conditions for traffic safety. Numerous constraints exist that are associated with providing low cost solutions by adopting off-the-shelf products. Many practical solutions have been developed, and numerous real-world tests have been conducted in this applied research. In this article, the focus is the performance of a small Mica2 Motes wireless sensor network as observed from a series of experiments in an outdoor environment using Surge Time Synchronization. Large transmitting range and long testing hours are among the features of the experimental investigation. The connectivity, packet reception rate, initial connection time, error rate and power consumption have been derived from test results and revealed the potential as well as challenges associated with this off-the-shelf product for real-world applications.

\section{ACKNOWLEDGMENTS}

This study is primarily sponsored by the Oklahoma Transportation Center under the OTC-22 project. A grant from the Research Council at the University of Oklahoma (OU) to initiate this project is also acknowledged. Dr Shivakumar Raman, the PI, and Ms Nina Diaz, the program coordinator, in the metrology Research Experience for Undergraduates (REU) program from the School of Industrial Engineering at OU are greatly appreciated for their support throughout the duration of this project. The technical assistance offered by Mr Michael F. Schmitz from the School of Civil Engineering \& Environmental Science (CEES) at OU throughout the project is deeply appreciated. A couple of technical discussions offered by Dr Venkatesh Saragan and Mr Jia (Winston) Liu from the Department of Computer Science at Oklahoma State University are greatly acknowledged for having greatly improved the understanding of the authors towards the inner-workings of Mica2 Motes. The assistance offered by Mr Yohanes P. Sugeng, Chase Sandburg, Oluwaseun Harris, Rory Victor, Olatunde Oshlake, Adam Heriba, Christopher A. Davis, and Ms Erin Hurt, is greatly acknowledged.

\section{REFERENCES}

Amphenol, R.F. 2003-2005. Mmcx 50 w straight cable connector with pin contact. http://www.amphenolrf.com/search_1_results. aspproduct type $=$ Connector\&impedance $=50 \&$ connector $\overline{\text { type }}=$ Cable\&connector $\quad$ series $=$ MMCX\&gender $=$ Pin\&body style $=$ Straight.

Anastasi, G., Conti, M., Falchi, A., Gregori, E. and Passarella. A., 2004. "Performance Measurements of Motes Sensor Networks," In: MSWiM 04: Proceedings of ACM, Venice, Italy, October 2004, pp. 174-181. Association for Computing Machinery, New York, NY 10036-5701, United States.

ANT-433-PW-QW Antenna Datasheet. 2006. Linx Technologies Inc., Oregon, USA.

Brewer, E., Culler, D., Gay, D., Levis, P., Von R., Behren, and Welsh, M. 2004. "nesc: A Programming Language for Deeply Networked Systems," Technical report, UC Berkeley WEBS Project, December 2004. 
Caffrey, J., Govindan, R., Johnson, E., Krishnamachari, B., Masri, S., Sukhatme, G., Chintalapudi, K., Dantu, K., Rangwala, S., Sridharan, A., Xu, N. and Zuniga. M. 2004. "Networked Sensing for Structural Health Monitoring," In: 4th International Workshop on Structrual Control, Columbia University, New York, NY, June 2004.

Cerpa, A., Wong, J.L., Potkonjak, M. and Estrin. D. 2005. “Temporal Properties of Low Power Wireless Links: Modeling and Implications on Multi-hop Routing," Technical report, Center for Embedded Networked Sensing, University of California at Los Angeles, January 2005.

Casciati, F., Faraveli, L. and Borghetti. F. 2003. "Wireless Links Between Sensor-device Control Stations in Long Span Bridges, In: Smart Structures and Materials 2003: Smart Systems and Nondestructive Evaluation for Civil Infrastructures, San Diego, CA, March 2003, pp. 1-7. Proceedings of SPIE Vol. 5057(2003). edited by S.-C. Liu.

Clayton, E., Koh, B., Xing, G., Fok, C., Dyke, S. and Lu. C. 2005. "Damage Detection and Correlation-based Localization using Wireless Mote Sensors," In: IEEE International Symposium on Intelligent Control - Proceedings, Vol. 1, Limassol, Cyprus, June 2005, pp. 304-309. IEEE Control Systems Society, CSS;Mediterranean Control Association, MCA, Institute of Electrical and Electronics Engineers Inc., Piscataway, NJ 08855-1331, United States.

Donoho. L. 2003. "Wireless Develops Extrasensory Vision of Future," Journal of New England Technology, http://www.bizjournals.com/ masshightech/stories/2003/01/13/focus $4 . h t m l$ (accessed on 10 January, 2003).

Ferzli, N.A., Sandburg, C.J., King, T., Pei, J.S., Zaman, M.M., Refai, H.H., Ivey, R.A. and Harris. O. 2006b. "Experimental Investigation of "Smart Dust" for Pavement Condition Monitoring" In: Proceedings of IMAC XXIV, Society for Experimental Mechanics, St. Louis, MO, January/February 2006 b.

Ferzli, N.A., Ivey, O., King, T., Sandburg, C.J., Pei, J.S., Zaman, M.M., Refai, H.H., Landrum, A.R. and Victor. R. 2006a. "An Application of "Smart Dust" for Pavement Condition Monitoring," In: Proceedings of SPIE Smart Structures and Materials and NDE for Health Monitoring and Diagnostics, San Diego, CA, SPIE. February/ March 2006a.

Glaser. S. 2004. "Some Real-world Applications of Wireless Sensor Nodes" In: Proceedings of SPIE - The International Society for Optical Engineering, Vol. 5391, San Diego, CA, March 2004, pp. 344-355, SPIE - The International Society for Optical Engineering; American Institute of Aeronautics and Astronautics, AIAA (USA);American Society of Mechanical Engineers, ASME (USA); Society for Experimental Mechanics, SEM (USA); The Boeing Company (USA), International Society for Optical Engineering, Bellingham, WA 98227-0010, United States.

GlobalSpec, 1999-2006. http://www.globalspec.com

Inc. Crossbow Technology. Wireless systems for environmental monitoring, 2006 http://www.xbow.com/Support/ Support_pdf_files/Smart Dust AppNote.pdf

Innovative Dynamics Inc. Icesight-wis, 2000-2006. http://www.idiny. com/icesightwis.html

Koushanfar F. and Potkonjak. M. 2005. "Markov Chain-based Models for Missing and Faulty Data in Mica2 Sensor Motes," In: 2005 IEEE Sensors, November 2005.

Kurata, N., Spencer Jr., B.F., Ruiz-Sandoval, M., Miyamoto, Y. and Sako. Y. 2003. "A Study on Building Risk Monitoring Using Wireless Sensor Network Mica Mote," In: Structural Health Monitoring and Intelligent Infrastructure, Tokyo, Japan, 13-15 November 2003, pp. 353-357. Proceedings of the First International Conference on Structural Health
Monitoring and Intelligent Infrastructure, edited by Z.S. Wu and M. Abe, Swets \& Zeitlinger B.V., Lisse, The Netherlands.

Leaf Wetness Sensor: Standard \& Industrial Installation Manual. Davis Instruments, 2002. Product Number: 6420.

Mahgoub, I. and Ilyas, M. 2006. Smart Dust: Sensor Network Application, Archietcture, and Design. CRC Press, Taylor \& Francis Group.

Mark J.W. and Zhuang. W. 2003. Wireless Communication and Networking. Prentice Hall.

Maurer. W. 2003. The Scientist and Engineer's Guide to TinyOS Programming, http://ttdp.org/tpg/html/book/book1.htm

Milligan. T.A., 2005. Modern Antenna Design, 2nd edn, Wiley-IEEE Press.

Mosalam, K.M., Machado, C., Glniorz, K.-U., Naito, C., Kunkel, E. and Mahin. S.A. 2002. "Seismic Evaluation of an Asymmetric Three-story Woodframe Building," Technical report, Consortium of Universities for Research in Earthquake Engineering (CUREE), December 2002.

NTC Thermistors, Accuracy Line. Vishay BCcomponents, 2322640 3/4/ 6.... Vishay, 2004. Document Number: 29049.

Paek, J., Chintalapudi, K., Caffrey, J., Govindan, R. and Masri. S. 2005. "A Wireless Sensor Network for Structural Health Monitoring: Performance and Experience," In: EmNet5-11, May 2005.

Patnode, D., Dunne, J., Malinowski, A. and Schertz. D. 2003. Wisenet tinyos Based Wireless Network of Sensors," In: IECON Proceedings Industrial Electronics Conference, Vol. 3, Roanoke, VA, November 2003, pp. 2363-2368. IEEE Industrial Electronics Society, Institute of Electrical and Electronics Engineers Computer Society.

Pei, J.S., Kapoor, C., Graves-Abe, T.L., Sugeng, Y.P. and Lynch, J.P. 2008. "An Experimental Investigation of the Data Delivery Performance of Wireless Sensing Units Designed for Structural Health Monitoring," Structural Control and Health Monitoring, Vol. 15, pp.471-504.

Pei, J.S., Ivey, R.A., Lin, H., Landrum, A.R., Sandburg, C.J., King, T., Ferzli, N.A., Zaman, M.M., Refai, H.H. and Mai. E.C. 2007. "Validation of Smart Dust Sensor Network for Pavement Condition Monitoring," Technical Report OTC-22, The Oklahoma Transportation Center.

QRD1113/1114 Reflective Object Sensor. Fairchild Seminconductor Corporation, 2000. Reference Number: 100030A.

Roadway Standard (English), Precast Median Barrier. Oklahoma Department of Transportation, 1999. Drawing Number: PMB-2.

Smart Dust Training, 2004. Crossbow Technologies Inc.

Shankar. P.M., 2002. Introduction to Wireless Systems, John Wiley.

The weather Channel Interactive, Inc., 2006 www.weather.com

Traffic Buttons. 2005. Trantex Inc.

UC Berkeley, 2004. http://www.tinyos.net/

Vaisala DRS511/DRS511B Road \&Runway Sensor. 2004a. Vaisala Inc.

Vaisala ROSA Weather Station. 2004b. Vaisala Inc.

Watertight Diecast Aluminum Small Enclosures. Hammond Manufacturing, 2006. Series 1590T (Basic).

Xu, N., Rangwala, S., Chintalapudi, K.K., Ganesan, D., Broad, A., Govindan, R. and Estrin. D. 2004. "A Wireless Sensor Network for Structural Monitoring," In: Proceedings of the 2nd International Conference on Embedded Networked Sensor Systems, Baltimore, MD, November 2004, pp. 13-24.

Zhao, J. and Govindan. R. 2003. "Understanding Packet Delivery Performance in Dense Wireless Sensor Networks," In: Proceedings of the 1st international conference on Embedded networked sensor systems, Los Angles, CA, November 2003, ACM Press, pp. 1-13. 